\title{
On the initial binary population for star cluster simulations
}

\author{
Diogo Belloni ${ }^{1,2 \star}$, Abbas Askar $^{1}$, Mirek Giersz $^{1}$, Pavel Kroupa $^{3,4}$ and \\ Helio J. Rocha-Pinto ${ }^{5}$ \\ ${ }^{1}$ Nicolaus Copernicus Astronomical Centre, Polish Academy of Sciences, ul. Bartycka 18, PL-00-716 Warsaw, Poland \\ ${ }^{2}$ CAPES Foundation, Ministry of Education of Brazil, DF 70040-020, Brasilia, Brazil \\ ${ }^{3}$ Helmholtz-Institut für Strahlen- und Kernphysik, Nussallee 1416, D-53115 Bonn, Germany \\ ${ }^{4}$ Charles University in Prague, Faculty of Mathematics and Physics, Astronomical Institute, V Holesovickach 2, CZ-180 00 Praha 8 , Czech Republic \\ ${ }^{5}$ Universidade Federal do Rio de Janeiro, Observatório do Valongo, Ladeira do Pedro Antônio 43, $20080-090$ Rio de Janeiro, Brazil
}

Accepted... . Received... ; in original form...

\begin{abstract}
Colour-magnitude diagrams (CMDs) are powerful tools that might be used to infer stellar properties in globular clusters (GCs), for example, the binary fraction and their mass ratio $(q)$ distribution. In the past few years, observations have revealed that q distributions of GC mainsequence (MS) binaries are generally flat, and a distribution characterized by a strong increase towards $\mathrm{q} \approx 1$ is not typical in GCs. In numerical simulations of GC evolution with the initial binary population (IBP) described by Kroupa, synthetic CMD colour distributions exhibit a peak associated with binaries that have $\mathrm{q} \approx 1$. While the Kroupa IBP reproduces binary properties in star-forming regions, clusters and the Galactic field, the peak in the q distribution towards $\mathrm{q} \approx 1$ observed for GC simulations is not consistent with distributions derived from observations. The objective of this paper is to refine and further improve the physical formulation of pre-main-sequence eigenevolution proposed by Kroupa in order to achieve CMD colour distributions of simulated GC models similar to those observed in real GCs, and to get a similarly good agreement with binary properties for late-type binaries in the Galactic field. We present in this paper a modified Kroupa IBP, in which early-type stars follow observational distributions, and late-type stars are generated according to slightly modified pre-main-sequence eigenevolution prescriptions. Our modifications not only lead to a qualitatively good agreement with respect to long-term observations of late-type binaries in the Galactic field, but also resolve the above-mentioned problem related to binary distributions in GC models.
\end{abstract}

Key words: methods: numerical - globular clusters: general - open clusters and associations: general - binaries: general

\section{INTRODUCTION}

The initial binary population (IBP) corresponds to initial properties of binaries in star clusters, which follow particular distributions of their parameters, i.e.primary mass, period, eccentricity, and mass ratio. The concept of an IBP is important because the full numerical solution to the problem of cloud collapse leading to star or binary formation is not viable (e.g. Kroupa 2011), but a description of initial populations is nevertheless needed for a wide variety of astrophysical problems - e.g. star cluster modelling, stellar population synthesis and dynamical population synthesis (e.g. Kouwenhoven et al. 2009).

It has been proposed that the field population of stars comes from the dissolution of star clusters (e.g. Lada \& Lada 2003) after the expulsion of the residual gas in the star formation process.

* E-mail: belloni@camk.edu.pl (DB)
Clustered star formation and dissolution of these embedded clusters via gas expulsion is the dominant process that populates the field with stars (e.g. Lada \& Lada 2003; Bressert et al. 2010; Lada 2010; Kroupa 2011; Marks \& Kroupa 2011). Additionally, it has been argued that the outcome of star-formation processes depends only weakly on the physical conditions of the molecular cloud (e.g. Kroupa 2011, and references therein). This is intrinsically associated with the IBP universality hypothesis which corresponds to an environment-independent star formation process (Marks et al. 2015, see their section 5.2.1).

Alternatively, some authors suggest that the star-formation process is not universal (e.g. King et al. 2012; Parker \& Meyer 2014). For example, King et al. (2012), on the basis of the comparison between the properties of close binary systems in seven young regions and in the field, conclude that the origin of multiplicity is not universal. In a similar way, Parker \& Meyer (2014) argue that the binary population in the field is indicative of the primordial bi- 


\section{Belloni et al.}

nary population in star-forming regions, at least for systems with primary masses in the range $0.02-3.0 \mathrm{M}_{\odot}$, based on comparisons between $N$-body simulations and binary properties in the Galactic field.

Concerning these two competing scenarios, we emphasize that both have the potential of explaining the observed data. However, as discussed in Marks et al. (2015), the hypothesis of the cluster origin of the galactic binary population in connection with the universality hypothesis is the only currently available approach that allows binary populations to be predicted in the Milky Way and other galaxies (Marks \& Kroupa 2011), and for such a reason this scenario is assumed in this work.

A promising candidate for the universal IBP has been inferred from observational data (Kroupa 1995b; Kroupa et al. 2013), hereafter called the Kroupa IBP. It has been tested against observations, and has successfully explained the observational features of young clusters, associations and Galactic field late-type binaries (e.g. Kroupa 2011; Marks \& Kroupa 2012). In addition, Belloni et al. (2017) found that models that follow the Kroupa IBP show good agreement with the observed Galactic cataclysmic variable white dwarf mass distribution. Direct imaging studies indeed reveal that most stars form in wide binaries (Sadavoy \& Stahler 2017).

Regarding the binary fraction in GCs, an efficient way of detecting the binary content is the search for binary mainsequences (MS) in colour-magnitude diagrams (CMDs) (e.g. Rubenstein \& Bailyn 1997). Sollima et al. (2007) were the first to investigate binaries in a reasonably large sample of $13 \mathrm{GCs}$, and found that all the analysed globular clusters contain a minimum binary fraction larger than 6 per cent within the core radius and that the global binary fractions lie between 10 to 50 per cent (depending on the cluster). Milone et al. (2012), who extended the sample and analysed MS binary populations in a sample of 59 GC CMDs, found for the first time an anti-correlation between the binary fraction and the total cluster mass.

Considering that, Leigh et al. (2015) investigated whether or not the observed present-day distribution of Galactic GC binary fractions can be reproduced assuming the Kroupa IBP (i.e. with a significant fraction of soft binaries). These authors showed that high initial binary fractions with a significant soft component (Kroupa IBP) combined with high initial densities can reproduce the observed anti-correlation between the binary fraction (both inside and outside the half-mass radius) and the total cluster mass, which corroborates the idea that an environmental independent universal IBP might exist associated with the binary population in the Galactic field, in associations, in young clusters and also in GCs.

Concerning the observed MS binary mass ratios in Galactic GCs, Milone et al. (2012) found that the distribution of the mass ratio is generally flat (for $q>0.5$ ), with the exception of only four GCs (E3, Terzan 7, NGC6366 and NGC6496). Since the MS binary mass ratio distribution is associated with the colour distribution on the red side of the fiducial MS, we should expect that such a distribution is also typically flat.

So far, no attempt to compare predicted and observed GC MS binary distributions has been carried out, taking into account the Kroupa IBP. On this regard, we will show in this work (Section 5) that, even though initial models following the Kroupa IBP predict present-day binary fractions supported by observations, there is a problem related to present-day MS binary distributions. In its original formalism, the Kroupa IBP contains a large fraction of binaries with approximately equal masses (i.e. $q \approx 1$, where $q$ is the mass ratio). This causes a clearly visible binary sequence close and above the MS turn-off in present-day GC CMD, and also colour distributions (below the turn-off) characterized by a strong increase towards the right edge of the distribution. Such features are, however, not commonly observed in GCs (Milone et al. 2012)

Rather than providing recipes for simulating present-day binary populations in GCs, the objective of this paper is first to show that the Kroupa IBP predicts present-day MS binary distributions (via analysis of present-day CMD colour distributions) which disagree with the observed ones, and second to develop a refined Kroupa IBP such that it can solve the above-mentioned problem with respect to GC MS binary distributions. Our modifications not only solve the problem, but also provide similar good agreement with Galactic field late-type binaries. In addition, this corroborates even more the universality hypothesis and provides a step forward in better descriptions for energy and angular momentum redistribution processes during star formation.

The paper is structured as follows. In Section 2 we define the main concepts and assumptions used in this investigation. In Section 3, we introduce the Kroupa IBP and in Section 5, we present the problem associated with the original prescription. The codes utilised in this investigation are described in Section 4, and a refined version which solves the problem with MS binary distributions is developed in Section 6. We show the main results with respect to binaries in the Galactic field and in GCs in Section 7. A summary of the main points of this investigation is provided in Section 8 .

\section{DEFINITIONS AND ASSUMPTIONS}

In order to clarify the terminology adopted in this work, in this section we define the main concepts explored and the main assumptions adopted. Note that the concept of IBP was already defined in Section 1 and it will be skipped in this section.

Kroupa (1995b) developed a simple model for the redistribution of energy and angular momentum in proto-binary systems such that it directly leads to binary properties (mass ratio, eccentricity and period) at the moment the star cluster dynamical evolution becomes effective. In practice, this process creates the IBP that should be used at the starting point of star cluster simulations. This process of converting birth binaries into initial binaries is usually called pre-main-sequence eigenevolution, since it occurs during the premain-sequence phase of binaries.

The first distinction we make is between the terms birth and initial binary populations. The term birth here is applied, as in Kroupa (1995b), to all protostars that are embedded in circumprotostellar material. On the other hand, the term initial corresponds to pre-main-sequence stars not embedded anymore in such a material, it being accreted or expelled during the process of redistribution of energy and angular momentum. We use the subscripts 'ini' and 'bir' to refer to the initial and birth population, respectively. The birth population can be viewed as a theoretical devise to allow calculations, and it is not observable because proto-binaries evolve rapidly on a time-scale of $10^{5} \mathrm{yr}$ in a deeply embedded phase. This is similar to the concept of initial mass function (i.e. the distribution of birth-stellar masses), which is not an observable distribution function and also does not exist, but it is an important tool for computations (Kroupa et al. 2013). With such a tool, predictions can be made for observable populations, which is why such theoretical distribution functions are needed.

The second distinction we consider is related to low-mass binaries and high-mass binaries. Low-mass binaries in this work correspond to all binaries whose primary mass is smaller than $5 \mathrm{M}_{\odot}$. 
Binaries with primary masses greater than this are called high-mass binaries. Note that we always assume that primaries are more massive than secondaries, and that mass ratios are always smaller than or equal to unity (i.e. $q=M_{2} / M_{1} \leqslant 1$ ).

The third distinction concerns short-period binaries and longperiod binaries. Short-period binaries have $P<10^{3}$ days while long-period binaries have $P \geqslant 10^{3}$ days.

Another distinction we make is related to the original Kroupa IBP and the modified Kroupa IBP. The original Kroupa IBP corresponds to the IBP generated through pre-main-sequence eigenevolution as described in Kroupa (1995b), for low-mass binaries and to the IBP described in Kroupa et al. (2013), for high-mass binaries. As we will see in Section 5, an inconsistency appears in simulated GC CMDs while comparing with real GC CMDs when the original Kroupa IBP is adopted. In order to overcome this problem, in this paper we propose a revised pre-main-sequence eigenevolution formulation, which leads in turn to a modified Kroupa IBP. Additionally, the modified Kroupa IBP, for high-mass binaries, assumes observed distributions for binaries whose primaries are $\mathrm{O}$ and $\mathrm{B}-$ dwarfs.

As a final concept, we use the term stimulated evolution to refer to the process of cluster dynamical evolution such that it converts the IBP to binaries whose distributions resemble those observed in the Galactic field. Usually, stimulated evolution is relatively fast and lasts for just a few Myr. In addition, residual gas removal due to the evolution of the most massive stars leading to significant cluster expansion (e.g. Brinkmann et al. 2017) is assumed to stop stimulated evolution.

As usually accepted, we assume that the Galactic field stellar population has its origin in clustered star formation (i.e. dynamical processing of binaries takes place before the dissolved cluster becomes part of the Galactic field. In addition, we assume that such clusters contain a high binary fraction (e.g. Duchêne 1999; Kroupa 2008). Actually, we assume $\approx 100$ per cent of binaries, which is consistent with resolving the angular momentum problem in star formation and the result that triples and higher order systems are rarely the outcome of late-type star formation (Goodwin \& Kroupa 2005), such that it leads to an appropriate binary fraction and binary properties for different spectral type stars after stimulated evolution.

\section{ORIGINAL KROUPA IBP}

In this section we describe the features of the original Kroupa IBP. These include properties of low-mass binaries (Section 3.1) and high-mass binaries (Section 3.2). In the end, in Section 5, we motivate the necessity of changing slightly the original prescriptions based on mock observations of present-day GC CMDs.

\subsection{Low-mass binaries (primary star mass $<5 \mathrm{M}_{\odot}$ )}

Note that the pre-main-sequence eigenevolution was developed in order to explain observational correlations found for G, K and M-dwarf binaries in the Galactic field (Duquennoy \& Mayor 1991; Mazeh et al. 1992; Fischer \& Marcy 1992), and was ultimately confirmed when data of all late-type binary systems near the Sun became available (Reid \& Gizis 1997). Therefore, pre-mainsequence eigenevolution is a process associated closely with latetype or low-mass binaries.

In what follows, we describe pre-main-sequence eigenevolution in its original formulation, i.e. Kroupa (1995b).

\subsubsection{Birth population}

In order to pass though pre-main-sequence eigenevolution, birth binaries are born with specific distributions, assumed here as:

i) Primary mass: randomly chosen from the Kroupa canonical initial mass function (IMF) (Kroupa et al. 1991).

ii) Secondary mass: secondary is randomly chosen from the same IMF (i.e. the mass ratio distribution is such that the binary components are randomly paired). Note that the primary and secondary are evident only after both stars are chosen independently from the canonical IMF.

iii) Eccentricity: it follows a thermal distribution, i.e.

$$
f_{e}=2 e .
$$

Here $\mathrm{d} N=f_{e} \mathrm{~d} e$ is the fraction of orbits with eccentricity in the range $e$ to $e+\mathrm{d} e$, amongst all orbits.

iv) Period: it follows Eq. 8 in Kroupa (1995b), i.e.

$$
f_{P}=2.5 \frac{\log _{10}(P / \text { days })-1}{45+\left[\log _{10}(P / \text { days })-1\right]^{2}} .
$$

Here $\mathrm{d} N=f_{P} \mathrm{~d} \log _{10} P$ is the fraction of orbits with period in the range $\log _{10} P$ to $\log _{10} P+\mathrm{d} \log _{10} P$ such that the integral of $f_{P}$ over all $\log _{10} P$ values equals the binary fraction of the stellar population, being one in this case.

During the pre-main-sequence eigenevolution phase, significant changes in the binary properties occur when the less massive object (secondary) is at the pericentre $\left(R_{p}\right)$, i.e., the distributions above (i-iv) applied for the birth population can be drastically changed during subsequent passages through the pericentre. A convenient way to calibrate the strength of the pre-main-sequence eigenevolution is by means of a function of $R_{p}$. This is expressed in the function $\rho$, which is defined as

$$
\rho=\left(\frac{\lambda \mathrm{R}_{\odot}}{R_{p}}\right)^{\chi}
$$

where $\lambda=28$ and $\chi=3 / 4$ (Kroupa 1995b), and $R_{p}$ is in units of $\mathrm{R}_{\odot}$. Note that the larger the pericentre distance, the smaller is the value of $\rho$.

\subsubsection{Initial population}

During the pre-main-sequence eigenevolution which has a duration of about $10^{5} \mathrm{yr}$, the birth population is converted to the initial population. The change in the eccentricity due to pre-mainsequence eigenevolution is given by

$$
\ln \left(e_{\text {ini }}\right)=-\rho+\ln \left(e_{\text {bir }}\right) .
$$

As during passages through the pericentre the secondary might accrete matter from the circumstellar disc around the primary, the changes in the mass ratio due to the pre-main-sequence eigenevolution is

$$
q_{\text {ini }}=\left\{\begin{array}{rll}
q_{\text {bir }}+\rho\left(1-q_{\text {bir }}\right), & \text { if } & \rho \leqslant 1 \\
1, & \text { if } & \rho>1
\end{array}\right.
$$

And the change of the secondary mass is given by

$$
M_{2, \text { ini }}=q_{\text {ini }} M_{1, \text { bir }} .
$$



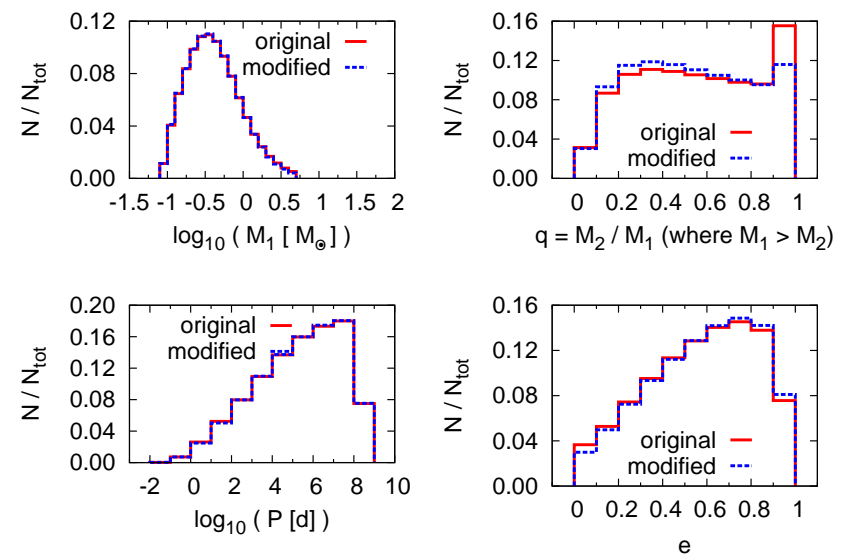

Figure 1. Primary mass (top left-hand panel), mass ratio (top right-hand panel), period (bottom left-hand panel), and eccentricity (bottom right-hand panel) distributions for all binaries such that $M_{1}<5 \mathrm{M}_{\odot}$ in the original Kroupa IBP (Section 3.1, red solid line) and in the modified Kroupa IBP (Section 6.2, blue dashed line).

Note that the primary mass remains unchanged during pre-main-sequence eigenevolution, i.e. $M_{1, \text { ini }}=M_{1 \text {,bir }}$ since the secondary unlikely has an appreciable circumstellar disk (Bonnell \& Bastien 1992).

Finally, the period after the pre-main-sequence eigenevolution is given by

$$
P_{\text {ini }}=P_{\text {bir }}\left(\frac{M_{1, \text { bir }}+M_{2, \text { bir }}}{M_{1, \text { ini }}+M_{2, \text { ini }}}\right)^{1 / 2}\left(\frac{1-e_{\text {bir }}}{1-e_{\text {ini }}}\right)^{3 / 2} .
$$

We must comment a few things at this point before proceeding further.

First, concerning mergers during the process of pre-mainsequence eigenevolution, binaries with pericentre distances greater than $1.1 \times\left(R_{1}+R_{2}\right)$ survive, and they merge otherwise, where $R_{1}$ and $R_{2}$ are the primary and secondary radii, respectively.

Second, the pre-main-sequence eigenevolution changes mainly short-period binaries since for these systems the function $\rho$ can be truly large. For long-period binaries, the effect of pre-mainsequence eigenevolution is inexpressive because the $\rho$ is extremely small (due to large pericentre distance) which leads to initial properties similar to birth properties.

Third, given the large amount of late-type pre-main-sequence objects generated according to the adopted IMF, many short-period low-mass binaries might have equal masses and low eccentricity due to pre-main-sequence eigenevolution, since they more easily have their properties changed. This has a huge impact in a star cluster population evolved over a Hubble time (Section 5) as these binaries are likely to survive for several Gyr of cluster dynamical evolution.

In Fig. 1 we plot the main distributions associated with the original Kroupa IBP regarding low-mass binaries, i.e. primary mass (top left-hand panel), mass ratio (top right-hand panel), period (bottom left-hand panel), and eccentricity (bottom right-hand panel). Note that we included as well the modified Kroupa IBP (Section 6.2) for comparison.
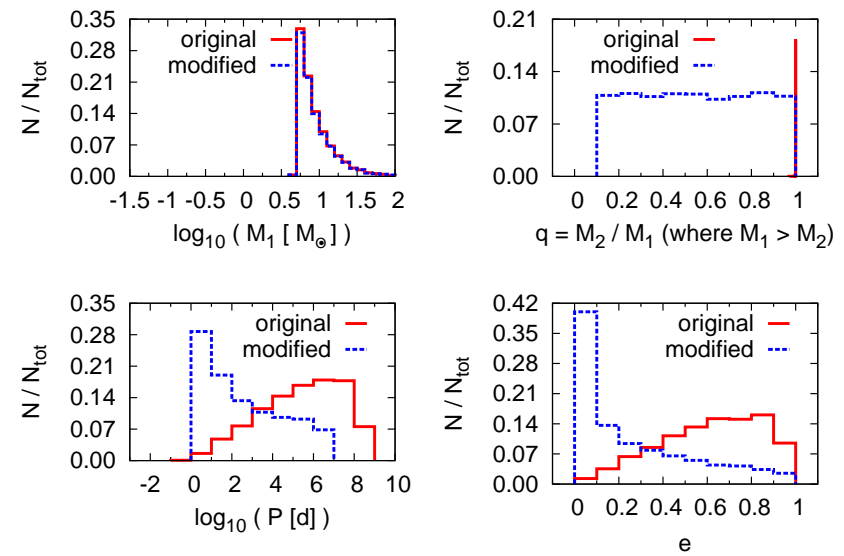

Figure 2. Primary mass (top left-hand panel), mass ratio (top right-hand panel), period (bottom left-hand panel), and eccentricity (bottom right-hand panel) distributions for all binaries such that $M_{1}>5 \mathrm{M}_{\odot}$ in the original Kroupa IBP (Section 3.2, red solid line) and in the modified Kroupa IBP (Section 6.3, blue dashed line).

\subsection{High-mass binaries (primary star mass $>5 \mathrm{M}_{\odot}$ )}

Since the pre-main-sequence eigenevolution theory was developed mainly to explain observed properties of Galactic field lowmass binaries (A, F, G, K and M-dwarfs), the question concerning how high-mass binaries $(\mathrm{O} \text { and } \mathrm{B} \text {-dwarfs })^{1}$ evolve remains open.

According to Kroupa et al. (2013), the initial properties of high-mass binaries follow precisely birth distributions, since premain-sequence eigenevolution is not applied in this range of masses. This is a reasonable assumption since for this range of masses, the timescale of pre-main-sequence evolution is short enough that it is safe to neglect it (Railton et al. 2014). Then, for high-mass binaries, birth and initial binaries follow distributions (iiv) in Section 3.1, with one exception, namely the secondary mass (i.e. mass ratio). Until very recently, observations of high-mass binaries have commonly revealed systems with nearly equal masses (e.g. Pinsonneault \& Stanek 2006; Mayer et al. 2017).

In order to account for this feature, in the original Kroupa IBP, a different pairing is usually adopted for high-mass binaries, namely ordered pairing (Kroupa et al. 2013). The procedure can be summarized as follows. Once all stars (twice the number of binaries) are generated according to the canonical Kroupa IMF, those with masses greater than $5 \mathrm{M}_{\odot}$ are subsequently ordered. The most massive star in the array is paired with the second most massive stars, and so on. This procedure guarantees that the IMF is preserved and generates high-mass binaries with similar masses, as previously thought from observational results.

Fig. 2 exhibits the main distributions associated with the original Kroupa IBP with respect to high-mass binaries, i.e. primary mass (top left-hand panel), mass ratio (top right-hand panel), period (bottom left-hand panel), and eccentricity (bottom right-hand panel). Note that we included as well the modified Kroupa IBP (Section 6.3) for comparison, as in Fig. 1.

1 Based on our definition for low-mass and high-mass binaries, binaries whose primaries are A-dwarfs are low-mass binaries, even though they are early-type binaries. We follow here the same threshold as in Kroupa et al. (2013). 


\section{NUMERICAL TOOLS}

We describe is this section the two codes used in this investigation for star cluster simulations (MOCCA, Section 4.1) and for the photometric reduction of mock observations of the simulated star clusters (COCOA, Section 4.2).

\subsection{The MOCCA code}

The MOcCA code (Hypki \& Giersz 2013; Giersz et al. 2013, and references therein) is based on the orbit-averaged Monte Carlo technique for cluster evolution developed by Hénon (1971), which was further improved by Stodółkiewicz (1986). It also includes the FEWBODY code, developed by Fregeau et al. (2004), to perform numerical scattering experiments of gravitational interactions. To model the Galactic potential, MOCCA assumes a point-mass with total mass equal to the enclosed Galaxy mass at the Galactocentric radius. The description of the escape processes in tidally limited clusters follows the procedure derived by Fukushige \& Heggie (2000). Stellar evolution is implemented via the SSE code developed by Hurley et al. (2000) for single stars and the BSE code developed by Hurley et al. (2002) for binary evolution.

MOCCA was extensively tested against $N$-body codes. For instance, Giersz et al. (2013) concluded that MOCCA is capable of reproducing $N$-body results with reasonable precision, not only for the rate of cluster evolution and the cluster mass distribution, but also for the detailed distributions of mass and binding energy of binaries. Additionally, Wang et al. (2016) also compared MOCCA with the state-of-the-art NBODY6++GPU and showed good agreement between the two codes. Finally, Madrid et al. (in prep.) showed that the MOCCA code is able to reproduce the escape rate from tidally limited clusters when compared with $N$-body codes, provided that they are not closer than a few kpc from the Galactic center.

\subsection{The COCOA code}

The COCOA code (Askar et al. 2017a) can create idealised mock observational data in the form of FITS files using numerical simulation snapshots at a specific time (provided by codes such as MOCCA/NBODY6/NBODY6++GPU). The COCOA code has been developed to extend results of numerical simulations of star clusters for the purpose of direct comparisons with observations. The input parameters in COCOA can be adjusted to create synthetic observations from virtually any optical telescope and the code can also carry out PSF photometry on the mock observations to create a catalogue of all observed stars in the cluster. These results can be used to observationally determine cluster parameters and create observational CMDs of a simulated star cluster model.

The COCOA code has already been used for creating mock observations using 12 Gyr simulation snapshots in investigations with the NBODY6++GPU code (Wang et al. 2016) and the MOCCA code (Askar et al. 2017b). COCOA has many different applications (Askar et al. 2017a) and can check if there are any systematics or biases associated with actual observational data and techniques used to determine cluster properties.

\section{PROBLEM WITH THE ORIGINAL KROUPA IBP}

The original Kroupa IBP (Section 3) has been tested against both numerical simulations and observations, and has successfully explained the observational features of young clusters, associations, Galactic field late-type binaries, and even binaries in old GCs (e.g. Kroupa 2011; Marks \& Kroupa 2012; Leigh et al. 2015, and references therein).

Even though the original prescription gives good results, there is at least one problem with the way pre-main-sequence eigenevolution changes the birth population. As noted in Section 3.1, the pre-main-sequence eigenevolution might be very strong for shortperiod G, K and M-dwarfs, in the sense that their initial properties can be extremely different from their birth properties. For example, substantial number of these short-period binaries will have equal masses and very low-eccentricities. This makes them very dynamically hard binaries which in turn allows them to survive in star clusters during long-term dynamical evolution (see Fig. 5).

The main implication of the above-mentioned result of the pre-main-sequence eigenevolution in low-mass short-period binaries in GCs are MS binary mass ratio distributions characterized by a significant fraction of binaries with $q \approx 1$, which are not typical in observed GCs (Milone et al. 2012).

First, in Section 5.1, we evolve 6 GC models (which follow initially the Kroupa IBP) with the MOCCA code and perform the photometric analysis in those models with the COCOA code. Then we show that the colour distributions in the synthetic CMDs, for all models, have conspicuous peaks towards the right edge of the distributions. Such peaks are associated with binaries whose mass ratios are $\approx 1$, as discussed above.

Second, in Section 5.2, we show CMDs of two real GCs and also their colour distributions, following the same procedure employed in the synthetic CMDs. We end this section by showing that the Kroupa IBP predicts colour distributions (and in turn MS binary mass ratio distributions) that are unlikely to exist in real GCs.

\subsection{GC models, synthetic CMDs and colour distributions}

In order to compare different synthetic CMDs with real CMDs, we evolved 6 models with different initial conditions for 12 Gyr. We adopt for all models the canonical IMF that follows the broken power law $\xi(m) \propto m^{-\alpha}$, defined by Kroupa et al. (2001), where $\alpha=1.3$ for $0.08 \leqslant m / \mathrm{M}_{\odot} \leqslant 0.5$ and $\alpha=2.3$ for $0.5 \leqslant m / \mathrm{M}_{\odot} \leqslant m_{\max } / \mathrm{M}_{\odot}$, and the star mass in this study lies between $0.08 \mathrm{M}_{\odot}$ and $100 \mathrm{M}_{\odot}$. Additionally, all models have 95 per cent of primordial binaries ${ }^{2}$. The metallicity equals $Z=0.001$, which is typical for GCs, and their binary properties are given by the original Kroupa IBP (Section 3).

We assume that all stars are on the zero-age main sequence when the simulation begins and that any residual gas from the star formation process has already been removed from the cluster. Additionally, all models are initially at virial equilibrium, and have neither rotation nor mass segregation. Moreover, all models are evolved for $12 \mathrm{Gyr}$ which is associated with the present-day in this investigation.

In Table 1, we summarize the main parameters of the initial and present-day models. Notice that we have a small set of GC models and, even being so, we still have very different initial and present-day properties, which guarantees that a considerable region of the parameter space is covered, allowing us in turn to access dif-

2 We set the initial binary fraction different from 100 per cent in order to avoid computational problems that arise in MOCCA if there is no single star in the initial model. 
Table 1: GC models, initial parameters that define them and present-day properties (i.e. after $12 \mathrm{Gyr}$ of evolution). The binary fraction, the metallicity and the the King model parameter are 95 per cent, 0.001 and 6.0 , respectively, for al models, In addition, for all models, we adopt the canonical Kroupa et al. (1991). IMF (i.e. with two segments), and the original Kroupa IBP (Section 3). The first column presents the model name and the following 9 columns give the initial values for the total mass (in $\mathrm{M}_{\odot}$ ), the total number of stars, the central density (in $\mathrm{M}_{\odot} \mathrm{pc}^{-3}$ ), the core radius (in pc), the half-mass radius (in pc), the tidal radius (in pc), the Galactocentric distance (in kpc), the half-mass radius relaxation time (in Myr), and the central velocity dispersion (in $\mathrm{km} \mathrm{s}^{-1}$ ). The remaining 11 columns provide present-day values for the total mass (in $\mathrm{M}_{\odot}$ ), the total number of stars, the central density (in $\mathrm{M}_{\odot} \mathrm{pc}^{-3}$ ), the core radius (in

4.1, for more details.

\begin{tabular}{|c|c|c|c|c|c|c|c|c|c|c|c|c|c|c|c|c|c|c|c|}
\hline \multicolumn{11}{|c|}{ initial } & \multicolumn{9}{|c|}{ present-day } \\
\hline Model & $\begin{array}{c}\mathrm{M} \\
{\left[\times 10^{5} \mathrm{M}_{\odot}\right]}\end{array}$ & $\begin{array}{c}\mathrm{N} \\
{\left[\times 10^{5}\right]}\end{array}$ & $\begin{array}{c}\rho_{c} \\
{\left[\mathrm{M}_{\odot} \mathrm{pc}^{-3}\right]}\end{array}$ & $\begin{array}{c}R_{\mathrm{c}} \\
{[\mathrm{pc}]}\end{array}$ & $\begin{array}{c}R_{\mathrm{h}} \\
{[\mathrm{pc}]}\end{array}$ & $\begin{array}{c}R_{\mathrm{t}} \\
{[\mathrm{pc}]}\end{array}$ & $\begin{array}{c}R_{\mathrm{G}} \\
{[\mathrm{kpc}]}\end{array}$ & $\begin{array}{c}T_{\mathrm{r}, \mathrm{h}} \\
{[\mathrm{Myr}]}\end{array}$ & $\begin{array}{c}\sigma_{c} \\
{\left[\mathrm{~km} \mathrm{~s}^{-1}\right]}\end{array}$ & $\begin{array}{c}\mathrm{M} \\
{\left[\times 10^{5} \mathrm{M}_{\odot}\right]}\end{array}$ & $\begin{array}{c}\mathrm{N} \\
{\left[\times 10^{5}\right]}\end{array}$ & $\begin{array}{c}\rho_{c} \\
{\left[\mathrm{M}_{\odot} \mathrm{pc}^{-3}\right]}\end{array}$ & $\begin{array}{c}R_{\mathrm{c}} \\
{[\mathrm{pc}]}\end{array}$ & $\begin{array}{l}R_{\mathrm{h}} \\
{[\mathrm{pc}]}\end{array}$ & $\begin{array}{c}R_{\mathrm{t}} \\
{[\mathrm{pc}]}\end{array}$ & $\begin{array}{c}T_{\mathrm{r}, \mathrm{h}} \\
{[\mathrm{Myr}]}\end{array}$ & $\begin{array}{c}\sigma_{c} \\
{\left[\mathrm{~km} \mathrm{~s}^{-1}\right]}\end{array}$ & Type $^{a}$ & $\begin{array}{c}\text { Total binary } \\
\text { fraction }\end{array}$ \\
\hline 1 & 3.70 & 7.8 & $2.43 \times 10^{4}$ & 0.71 & 2.4 & 60 & 4.4 & $5.60 \times 10^{2}$ & 25.2 & 2.07 & 6.2 & $3.6 \times 10^{7}$ & 0.38 & 5.6 & 49.4 & $3.5 \times 10^{3}$ & 34.5 & cIMBH & 0.12 \\
\hline 2 & 3.70 & 7.8 & $4.87 \times 10^{2}$ & 2.62 & 8.8 & 60 & 4.4 & $3.95 \times 10^{3}$ & 13.2 & 1.06 & 3.0 & $1.9 \times 10^{2}$ & 1.81 & 8.1 & 39.2 & $4.2 \times 10^{3}$ & 6.1 & $\mathrm{pc}$ & 0.24 \\
\hline 3 & 3.70 & 7.8 & $3.89 \times 10^{3}$ & 1.31 & 4.4 & 30 & 1.6 & $1.40 \times 10^{3}$ & 18.6 & 0.09 & 0.2 & $2.4 \times 10^{5}$ & 0.02 & 1.4 & 8.9 & $7.2 \times 10^{1}$ & 4.5 & c & 0.30 \\
\hline 4 & 9.25 & 19.5 & $1.05 \times 10^{5}$ & 0.74 & 2.4 & 60 & 2.8 & $7.99 \times 10^{2}$ & 30.7 & 5.55 & 16.9 & $5.8 \times 10^{8}$ & 0.58 & 4.9 & 50.6 & $4.6 \times 10^{3}$ & 45.7 & cIMBH & 0.09 \\
\hline 5 & 9.25 & 19.5 & $2.11 \times 10^{3}$ & 2.73 & 8.8 & 60 & 2.8 & $5.64 \times 10^{3}$ & 16.0 & 2.91 & 8.5 & $3.6 \times 10^{2}$ & 2.53 & 8.6 & 40.8 & $7.1 \times 10^{3}$ & 10.1 & $\mathrm{pc}$ & 0.20 \\
\hline 6 & 9.25 & 19.5 & $1.69 \times 10^{4}$ & 1.37 & 4.4 & 30 & 1.0 & $1.99 \times 10^{3}$ & 22.7 & 1.15 & 2.5 & $3.3 \times 10^{4}$ & 0.19 & 2.4 & 14.9 & $5.8 \times 10^{2}$ & 11.0 & $\mathrm{pc}$ & 0.16 \\
\hline
\end{tabular}

${ }^{a}$ The cluster present-day type can be: post-core collapse (c), post-core collapse with intermediate-mass black hole (cIMBH) and pre-core collapse (pc).

Table 2: GC models (whose initial conditions are exactly the same as those listed in Table 1), having initial binaries following the modified Kroupa IBP (Section 6). Columns are the same as in Table 1. Notice that model 4, which contains a very massive IMBH (which dominates the dynamics of the core), has a significantly larger central velocity dispersion, when compared with the same model in Table 1 . This is because the process of IMBH formation is stochastic (Giersz et al. 2015), so the masses of IMBHs at $12 \mathrm{Gyr}$ are different, which causes differences in non-mass-weighted central velocity dispersions. See Section 4.1, for more details.

\begin{tabular}{|c|c|c|c|c|c|c|c|c|c|c|c|c|c|c|c|c|c|c|c|}
\hline \multicolumn{11}{|c|}{ initial } & \multicolumn{9}{|c|}{ present-day } \\
\hline Model & $\begin{array}{c}\mathrm{M} \\
{\left[\times 10^{5} \mathrm{M}_{\odot}\right]}\end{array}$ & $\begin{array}{c}\mathrm{N} \\
{\left[\times 10^{5}\right]}\end{array}$ & $\begin{array}{c}\rho_{c} \\
{\left[\mathrm{M}_{\odot} \mathrm{pc}^{-3}\right]}\end{array}$ & $\begin{array}{c}R_{\mathrm{c}} \\
{[\mathrm{pc}]}\end{array}$ & $\begin{array}{l}R_{\mathrm{h}} \\
{[\mathrm{pc}]}\end{array}$ & $\begin{array}{c}R_{\mathrm{t}} \\
{[\mathrm{pc}]}\end{array}$ & $\begin{array}{c}R_{\mathrm{G}} \\
{[\mathrm{kpc}]}\end{array}$ & $\begin{array}{c}T_{\mathrm{r}, \mathrm{h}} \\
{[\mathrm{Myr}]}\end{array}$ & $\begin{array}{c}\sigma_{c} \\
{\left[\mathrm{~km} \mathrm{~s}^{-1}\right]}\end{array}$ & $\begin{array}{c}\mathrm{M} \\
{\left[\times 10^{5} \mathrm{M}_{\odot}\right]}\end{array}$ & $\left.\begin{array}{c}\mathrm{N} \\
{\left[\times 10^{5}\right.}\end{array}\right]$ & $\begin{array}{c}\rho_{c} \\
{\left[\mathrm{M}_{\odot} \mathrm{pc}^{-3}\right]}\end{array}$ & $\begin{array}{c}R_{\mathrm{c}} \\
{[\mathrm{pc}]}\end{array}$ & $\begin{array}{l}R_{\mathrm{h}} \\
{[\mathrm{pc}]}\end{array}$ & $\begin{array}{c}R_{\mathrm{t}} \\
{[\mathrm{pc}]}\end{array}$ & $\begin{array}{c}T_{\mathrm{r}, \mathrm{h}} \\
{[\mathrm{Myr}]}\end{array}$ & $\begin{array}{c}\sigma_{c} \\
{\left[\mathrm{~km} \mathrm{~s}^{-1}\right]}\end{array}$ & Type a & $\begin{array}{l}\text { Total binary } \\
\text { fraction }\end{array}$ \\
\hline 1 & 3.70 & 7.8 & $2.43 \times 10^{4}$ & 0.71 & 2.4 & 60 & 4.4 & $5.60 \times 10^{2}$ & 25.2 & 2.05 & 6.24 & $\overline{5.4 \times 10^{7}}$ & 0.21 & 5.5 & 499.4 & $3.5 \times 10^{3}$ & 33.8 & cIMBH & 0.12 \\
\hline 2 & 3.70 & 7.8 & $4.87 \times 10^{2}$ & 2.62 & 8.8 & 60 & 4.4 & $3.95 \times 10^{3}$ & 13.2 & 1.04 & 2.99 & $6.8 \times 10^{2}$ & 3.62 & 8.1 & 39.5 & $4.2 \times 10^{3}$ & 6.0 & $\mathrm{pc}$ & 0.23 \\
\hline 3 & 3.70 & 7.8 & $3.89 \times 10^{3}$ & 1.31 & 4.4 & 30 & 1.6 & $1.40 \times 10^{3}$ & 18.6 & 0.09 & 0.16 & $4.3 \times 10^{5}$ & 0.05 & 1.3 & 8.9 & $6.9 \times 10^{1}$ & 4.7 & $\mathrm{c}$ & 0.26 \\
\hline 4 & 9.25 & 19.5 & $1.05 \times 10^{5}$ & 0.74 & 2.4 & 60 & 2.8 & $7.99 \times 10^{2}$ & 30.7 & 5.48 & 16.98 & $8.6 \times 10^{8}$ & 0.36 & 4.9 & 50.6 & $4.6 \times 10^{3}$ & 66.9 & cIMBH & 0.08 \\
\hline 5 & 9.25 & 19.5 & $2.11 \times 10^{3}$ & 2.73 & 8.8 & 60 & 2.8 & $5.64 \times 10^{3}$ & 16.0 & 2.76 & 8.22 & $2.5 \times 10^{3}$ & 3.28 & 8.5 & 40.3 & $7.0 \times 10^{3}$ & 9.6 & $\mathrm{pc}$ & 0.20 \\
\hline 6 & 9.25 & 19.5 & $1.69 \times 10^{4}$ & 1.37 & 4.4 & 30 & 1.0 & $1.99 \times 10^{3}$ & 22.7 & 1.04 & 2.25 & $2.5 \times 10^{4}$ & 0.32 & 2.3 & 14.5 & $5.3 \times 10^{2}$ & 10.8 & $\mathrm{pc}$ & 0.16 \\
\hline
\end{tabular}

${ }^{a}$ The cluster present-day type can be: post-core collapse (c), post-core collapse with intermediate-mass black hole (cIMBH) and pre-core collapse (pc). 
(1)

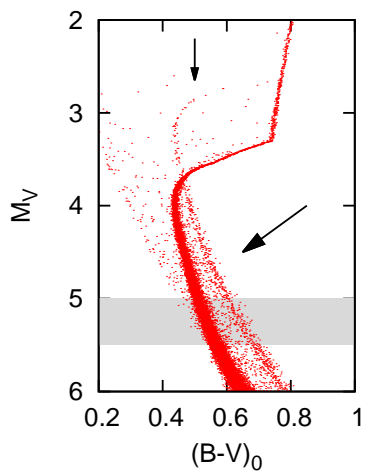

(3)

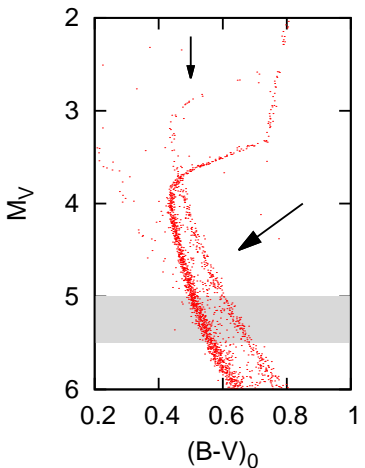

(5)

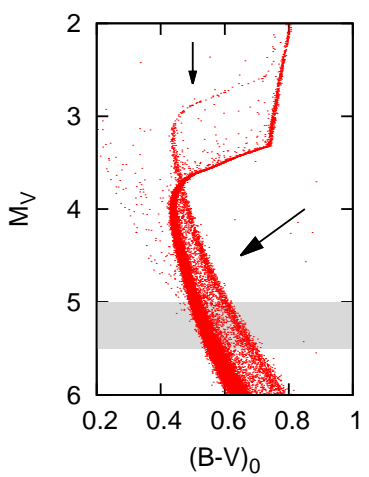

(2)

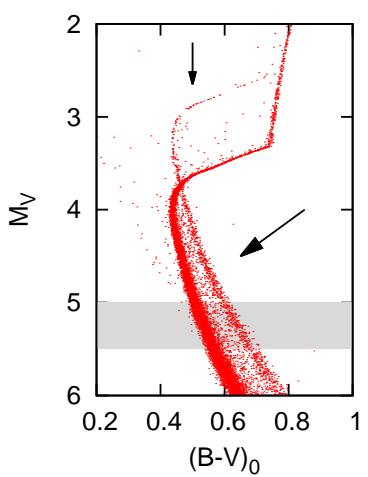

(4)

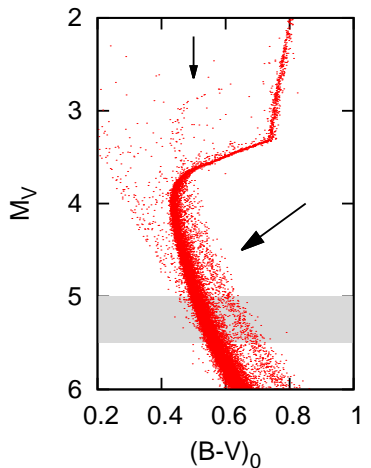

(6)

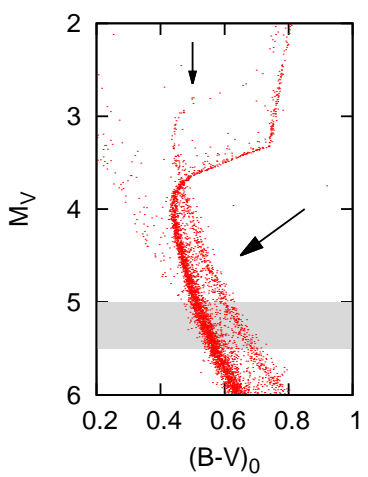

Figure 3. Present-day CMDs from simulated observations of the six GC models (Table 1) evolved with the original Kroupa IBP (Section 3). Regions used to generate colour distributions in Fig. 4 are demarcated by gray areas. Note that there is a pronounced binary sequence (particularly above the MS turn-off ) due to short-period low-mass binaries with mass ratios of unity in the model that has been indicated by the black arrows in the figure. For more detail see Section 5.1.

ferent CMD morphologies arising from different choices of initial conditions.

In order to investigate the distribution of binaries in GC models simulated with the original Kroupa IBP (Section 3), CMDs were obtained by imaging and carrying out photometry on the inner parts of the clusters (within their half-light radii) with the COCOA code, assuming that the clusters are at a heliocentric distance of $5 \mathrm{kpc}$. The observations were simulated with an HST type telescope with
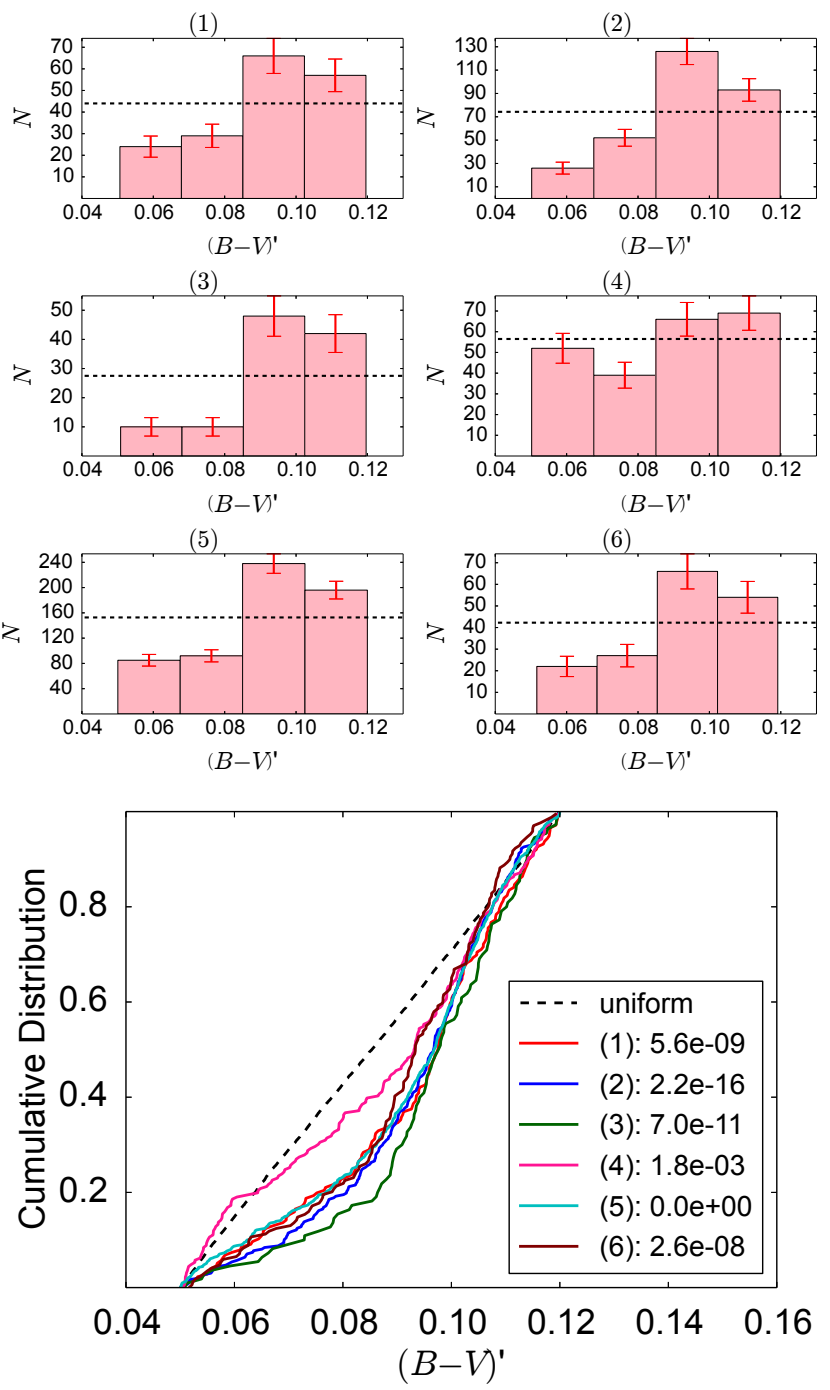

Figure 4. Colour distributions derived from the CMDs shown in Fig. 3, following the procedure described in Section 5.1. In the first six panels, we show histograms together with horizontal lines which are average numbers, assuming flat distributions. Vertical lines in the histograms correspond to Poisson errors. In the last panel, we compare cumulative distributions, and display in the key $p$-values of one-sample Kolmogorov-Smirnov tests for uniformity, for each model. Only stars with $(B-V)^{\prime}>0.05$ are used to compute the above distributions. Note that in all colour distributions a conspicuous peak is observed towards the right edge the distributions, which is associated with the binary sequences visible in the CMDs exhibited in Fig. 3. With respect to the cumulative distributions, it is clear that the colour distributions in the six models following the original Kroupa IBP are not uniform. This is also supported by the statistical test, which allows us to reject the hypothesis that they are uniform with more than $99 \%$ of confidence (see $p$-values). For more detail see Sections 5.1.

a pixel scale of $0.05 \% /$ pix, a seeing value ${ }^{3}$ in both the filters (B and V) of 0.15 ", and a Gaussian PSF was used. While these idealized mock observations do not model the exact HST PSF, the combination of high spatial resolution images with extremely low seeing

3 We emphasize that with HST-like observations we should have no seeing, a priori. However, in order to perform photometry, we need to achieve a good full width at half maximum (FWHM) value, which implies that a definition of seeing is required. 


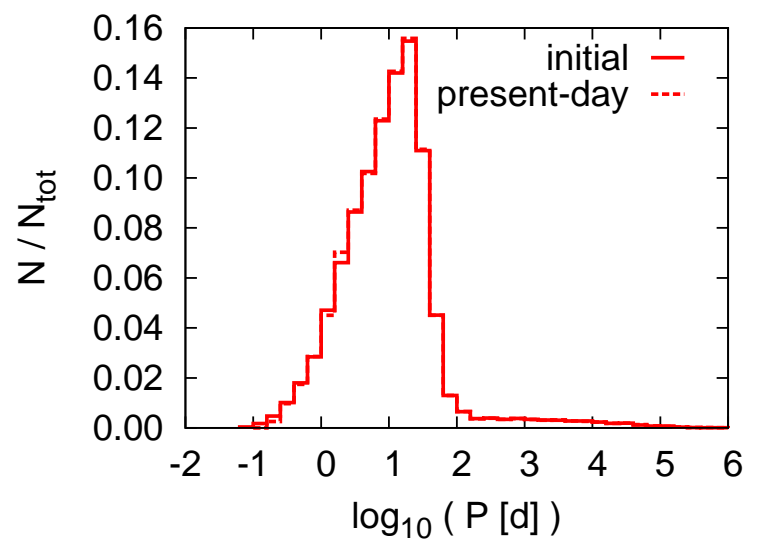

Figure 5. Initial and present-day period distributions of all MS binaries whose $q>0.99$, in all six models described in Table 1 . Note that both distributions are practically the same, which strongly supports the idea that the binary sequence for binaries with $q \approx 1$ comes from primordial binaries practically not affected by dynamics. The reason is because such binaries are very-short-period, having the majority of binaries with $q \approx 1$ periods shorter than 100 days. This suggests that in order to avoid the prediction of such binary sequences, the IPB has to be changed. For more detail see Sections 5.1 .

values can reproduce FWHM values for HST images in optical filters. The PSF photometry obtained from these synthetic images are comparable to the results from HST photometry particularly for the magnitude regime that we are interested in.

From the synthetic CMDs, we obtained the fiducial MS in the following way, which is similar to the procedure adopted in Milone et al. (2012). First we select all stars in the magnitude interval of interest, which is adopted here as $[5.0,5.5]$. Second we divide such an interval into 40 bins and obtain the median (in colour and magnitude) in the $2 \mathrm{D}$ region defined by each interval. Finally the fiducial MS, in the interval, has been derived by fitting these median points with a line. From such a fiducial MS, we have the line properties, which allows us to rotate and translate this region such that the MS becomes a vertical line centred at the origin of the $\mathrm{x}$ axis (the colour axis). From this rotated and translated part of each CMD, we compute the rotated colour $(B-V)^{\prime}$ distribution, starting from the value $(B-V)^{\prime}=0.05$. This lower limit guarantees that the systems, for $(B-V)^{\prime}>0.05$, are likely binaries with $q>0.5$. System with $(B-V)^{\prime}<0.05$ are either single stars belonging to the MS or binaries close to the MS (i.e. binaries with $q<0.5)$.

Fig. 3 exhibits the synthetic CMDs and Fig. 4 the colour distributions. Notice that the colour distribution is an increasing function of the colour and that the peaks shown in the right edge of each distribution are directly connected with the abundant presence of MS binaries whose mass ratios are $\approx 1$. The large width of the peak is connected with observational errors, which are larger for less luminous stars.

From the histograms in Fig. 4 we can expect that the distributions are not uniform, since the dashed horizontal lines (average number, assuming a uniform colour distribution) are unlikely good fits for the histograms. In order to confirm this, we applied a onesample Kolmogorov-Smirnov test for uniformity to the six models. All models exhibit rather small $p$-values (see keys in bottom panel of Fig. 4). The result of this test allows rejection of the null hypothesis that the colour distributions are uniform with more than 99
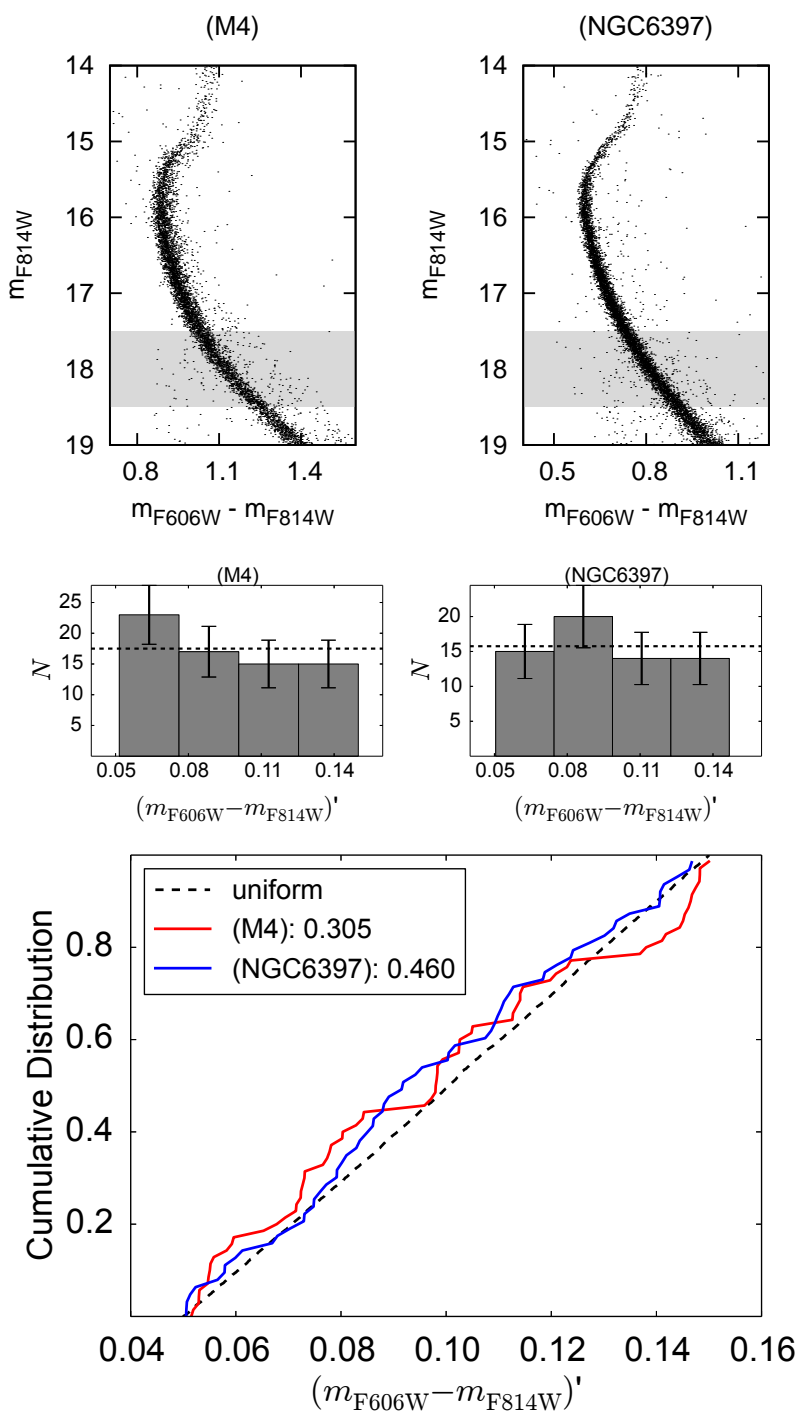

Figure 6. CMD (top panels) and colour distributions (middle and bottom panels) of M4 (left-hand column) and NGC6397 (right-hand column). The CMDs were generated with the HST Globular Cluster Treasury catalogue (Sarajedini et al. 2007), which is based on HST ACS/WFC data, and the colour distributions were generated following the prescription in Section 5.1. Only stars with $\left(m_{\mathrm{F} 606 \mathrm{~W}}-m_{\mathrm{F} 814 \mathrm{~W}}\right)^{\prime}>0.05$ are used to compute the above distributions. The gray areas in the CMDs indicate the region from where the colour distributions were derived, and the horizontal lines in the histograms are average numbers, assuming flat distributions. Notice that in these two GCs, taken as examples because of their proximities, pronounced sequences caused by binaries with $q \approx 1$ are not visible, especially in the region above and close to the turn-off (compare with Fig. 3). Additionally, their colour distributions are consistent with uniform distributions. This is is indicated in the last panel which shows the colour cumulative distributions and the $p$-values of one-sample Kolmogorov-Smirnov tests for uniformity. The test does not allow us to reject the hypothesis that they are uniform. For more detail see Sections 5.2.

per cent of confidence. This indicates that, if MS binary mass ratio distributions (and in turn colour distributions) in real GCs are consistent with uniformity, then a potential problem with the Kroupa IBP in matching binary distributions in GCs seems to exist.

In order to show that the peaks in the colour distribution in Fig. 4 are instrinsically connected with the Kroupa IBP, we show 
in in Fig. 5 the initial and present-day period distributions of all present-day MS binaries whose $q>0.99$ in all six models. We note that both distributions are basically the same, which supports that they are primordial binaries not strongly affected by dynamics. In addition, the majority of these binaries have periods shorter than 100 days, which makes them very dynamically hard and not easily subject to disruption via dynamical interactions.

In the following section, we show, as examples, two real GC CMDs and their colour distributions, compare them with results obtained for the original Kroupa IBP, and conclude that the original Kroupa IBP is probably not good in matching MS binary distributions in real GCs.

\subsection{Real GC CMDs and the problem with the original Kroupa IBP}

In order to show that GC models set with the original Kroupa IBP are not good to reconcile observed CMD morphologies and MS binary colour distributions, we analyse here two real GCs, namely M4 and NGC6397. These two clusters are the nearest GCs (Harris 1996, 2010 edition) and are also therefore well-studied. In addition to the quality of data concerning these two GCs, M4 can be described by a classic King profile, while NGC6397 is a post-core collapse cluster, which allows us to investigate CMDs and colour distributions in two different 'dynamical regimes'.

Fig. 6 exhibits the CMDs and the colour distributions of M4 and NGC6397. The CMDs were generated with the HST Globular Cluster Treasury catalogue (Sarajedini et al. 2007), which is based on HST ACS/WFC data, and the colour distributions were generated following the prescription in Section 5.1, being the magnitude interval $[17.5,18.5]$ in both GCs.

The first incompatibility we notice while comparing Figs. 4 and 6 is the absence of a pronounced binary sequence above the MS turn-off in the CMDs of M4 and NGC6397. This sort of sequence is present in all CMDs of Fig. 4, which is caused by binaries which are very dynamically hard and have $q \approx 1$, as illustrated in Figs. 4 and 5. Additionally, if this feature is present in all CMDs, and if the original Kroupa IBP should correspond to initial GC binary properties, then such a behaviour should be also visible in real GCs. Even in the nearest GCs this is not the case, which implies that the original Kroupa IBP seems not to be adequate to reproduce binary distributions in real GCs.

Similarly, contrary to what is predicted when models are set with the original Kroupa IBP, the colour distributions do seem to be flat in real GCs, since the dashed horizontal lines in the histograms appear to be a good parent model for the distributions. Again, in order to test this in a more coherent way, we applied one-sample Kolmogorov-Smirnov tests for uniformity. The $p$-values are 0.305 and 0.46, for M4 and NGC6397, respectively. This test does not allow us to reject the hypothesis that their colour distributions are uniform, which implies that they are consistent with uniform distributions.

We stress here that we decided to perform the analysis with respect to only two GCs because several investigations have already been carried out with regards to CMDs obtained with HST data (e.g. Piotto et al. 2002; Sarajedini et al. 2007; Milone et al. 2012). None of them is consistent with a mass ratio distribution peaked at $q \approx 1$. In addition, since Milone et al. (2012) have already concluded that the observed MS mass ratio distributions in GCs are consistent with uniform distributions, we expect that our procedure concerning the colour distributions would reveal the same trends found for M4 and NGC6397 (i.e. colour distribution consistent with the hypothesis of uniformity), if more GCs were included.

Additionally, the HST F606W and F814W photometric filter bands approximately correspond to the $\mathrm{V}$ and I band filters, respectively. So our use of the observational CMDs in terms of (V-I) vs I are a very good approximation to HST data sufficient for the present purpose. Askar et al. (2017a) have shown that such a binary sequence is also very pronounced (see their figs. 11, 20 and 21) in CMDs generated with the $\mathrm{V}, \mathrm{U}$ and I bands. Therefore, there should not be any serious biases in the compared data sets, in particular, since we are not aiming at a detailed modelling of a particular cluster.

Summarising, binary distributions found in present-day GC models set with the original Kroupa IBP are unlikely to match those derived from observations and the evidence for that is twofold. First in part of the CMD above the MS turn-off, a pronounced binary sequence is clearly predicted, although not observed. Second, the observed colour distributions below the turn-off are consistent with uniform distributions, and predicted distributions are characterized by a peak in the right edge of the distributions. As both discrepancies are mainly caused by the presence of a significant fraction of short-period low-mass binaries whose component masses are similar, a way to avoid such a non-observed effect is to reduce the fraction of these systems in the IBP. In what follows, we describe how we did this by changing slightly the pre-main-sequence eigenevolution prescription as well as what distributions we adopt for highmass binaries, which differ drastically from those of the original Kroupa IBP.

\section{MODIFIED KROUPA IBP}

In order to prevent the discrepancies pointed out in the previous section with respect to MS binary distributions (due to binaries whose components have equal masses) in present-day GC CMDs, we can change the pre-main-sequence eigenevolution prescription. In what follows, we describe the procedure to find a revised premain-sequence eigenevolution process as well as what sort of distributions for high-mass binaries we should adopt in order to respect observational constraints.

\subsection{Looking for a revised pre-main-sequence eigenevolution}

The first step we adopted in changing the pre-main-sequence eigenevolution is the strength with which the secondary accretes matter from the circumstellar disk around the primary. This is intrinsically associated with one parameter in Section 3.1, namely $\lambda$ (Eq. 3) and with the efficiency of the accretion expressed by $\rho$ (Eq. $5)$.

In order to reduce such a strength of increasing the secondary mass, we assumed a mass-depend $\lambda$ such that the lower the primary mass, the weaker the redistribution of energy and angular momentum within their circumstellar material. This dependence with the primary mass would take into account differences in binaries with different spectral types. Thus, we assumed that $\lambda\left(M_{1}\right)$ is a monotonic function of the primary mass up to a critical primary mass $M_{\text {crit }}$ and a constant after that, i.e.

$$
\lambda\left(M_{1}\right)=\left\{\begin{array}{rll}
\lambda_{0} \times M_{1}^{\eta}, & \text { if } & M_{1} \leqslant M_{\text {crit }}, \\
\lambda_{0}, & \text { if } & M_{\text {crit }}<M_{1}<5 \mathrm{M}_{\odot},
\end{array}\right.
$$

where $\lambda_{0}, \eta$, and $M_{\text {crit }}$ are parameters to be determined. 
In addition to a mass-dependent $\lambda\left(M_{1}\right)$, we also added a parameter $\varepsilon \in(0,1)$ in Eq. 5 (associated with the efficiency at which the secondary mass accretes matter from the primary circumstellar disk). We also added in the expression a stochastic component in the accretion process of the secondary for low-mass binaries whose primary masses are smaller than $M_{\text {crit }}$. The modified secondary accretion process, i.e. modified initial mass ratio is given by

$q_{\text {ini }}=\left\{\begin{aligned} q_{\text {bir }}+\rho_{0}\left(1-q_{\text {bir }}\right), & \text { if } \quad \rho_{0}<1, \\ 0.9+0.1 \times U(0,1), & \text { if } \quad \rho_{0} \geqslant 1 \text { and } M_{1}<M_{\text {crit }}, \\ 1, & \text { if } \quad \rho_{0} \geqslant 1 \text { and } M_{1} \geqslant M_{\text {crit }},\end{aligned}\right.$

where $U(0,1)$ is the standard uniform distribution and

$$
\rho_{0}=\left\{\begin{array}{cll}
\varepsilon \rho, & \text { if } M_{1}<M_{\text {crit }}, \\
\rho, & \text { if } M_{1} \geqslant M_{\text {crit }} .
\end{array}\right.
$$

Note that in the original Kroupa IBP, $\varepsilon=1$ by construction. Since we aim to reduce the fraction of short-period low-mass binaries with equal mass components, we allowed $\varepsilon$ (efficiency of secondary mass increase) to be smaller than unity, its value heeding to be determined as for $\lambda_{0}, \eta$, and $M_{\text {crit }}$. This is a reasonable assumption if one realises that the change in the eccentricity need not be coupled with the change in the secondary mass. They are in principle two distinct physical process. In fact, as we will see, the efficiency for changing the eccentricity is different from that associated with the changes in the secondary mass.

Note also that we introduced a random initial mass ratio in the range of $(0.9,1.0)$, when $\rho_{0}$ is greater than unity and $M_{1}$ is less than $M_{\text {crit }}$. This was done to account for a stochastic component in the accretion process of the secondary. Again, this improvement was motivated by present-day CMDs which exhibit pronounced high-q binary sequences caused by G-dwarfs and K-dwarfs with mass ratio near 1.0. With such a modification, short-period lowmass binaries unlikely have components with nearly the same mass.

Finally, note that in this procedure, for convenience, we kept $\chi$ in Eq. 3 fixed and given by $3 / 4$ as in the original Kroupa IBP. We calculated more than 200 models varying conveniently the abovementioned parameters with the following values

- $\lambda_{0}: 20,28,36$,

- $M_{\text {crit }}: 1.0,1.5,2.0$,

- $\eta$ : $0.25,0.50,0.75,1.00,1.25,1.50,1.75,2.00$,

- $\varepsilon$ : $0.25,0.30,0.50$.

Given each model, we used properties of late-type binaries that are not strongly affected by dynamics, mainly massratio and eccentricity distribution of short-period binaries, to compare with observational data of G-dwarf binaries in the Galaxy (Duquennoy \& Mayor 1991), using the minimum $\chi^{2}$ method of model-fitting. Our best-fit model has the following values:

$$
\begin{cases}\lambda_{0} & =28, \\ M_{\text {crit }} & =1 \mathrm{M}_{\odot}, \\ \eta & =1 / 4, \\ \varepsilon & =1 / 2 .\end{cases}
$$

Note that $M_{\text {crit }}=1 \mathrm{M}_{\odot}$ and $\lambda_{0}=28$ which implies that our modifications are effective only for G, K and M-dwarfs. For the rest of the low-mass binaries, the modified Kroupa IBP is exactly the same as the original Kroupa IBP. Note also that $\varepsilon=1 / 2$ which implies that the efficiency in the change of the mass ratio is half in comparison with the change in the eccentricity, for bina- ries whose primary mass is smaller than $M_{\text {crit }}=1 \mathrm{M}_{\odot}$. Finally, since $\lambda_{0}=28$ and $\eta=1 / 4$, the $\rho$ as defined in Eqs. 3 and 8 is a smooth, continuous and monotonically non-decreasing function of the primary mass $M_{1}$, in the range where pre-main-sequence eigenevolution should be applied.

In Section 7 we show the results for the modified Kroupa IBP that will be described in the following sections.

\subsection{Low-mass binaries}

To obtain the initial population for low-mass binaries in our revised pre-main-sequence eigenevolution procedure, we follow exactly the same approach as that to generate the original Kroupa IBP. The only difference in the modified Kroupa IBP is the different equations that are used.

We first generate the birth population with distributions (i-iv) in Section 3.1.1. Then we convert the birth population to the initial population using Eqs. 8, 3, 4, 9, and 7, with the parameter values given by Eq. 11 .

In Fig. 1 we plot the main distributions associated with the modified Kroupa IBP for low-mass binaries, i.e. primary mass (top left-hand panel), mass ratio (top right-hand panel), period (bottom left-hand panel), and eccentricity (bottom right-hand panel). Note that the original Kroupa IBP (Section 3.1) was included for comparison.

Note that the main difference between the original and the modified Kroupa IBPs is the mass ratio distribution. Indeed, in the modified Kroupa IBP the fraction of binaries in the range of $q \in[0.9,1.0]$ is reduced due to the revised $\lambda$ (Eq. 8) and the revised secondary accretion process (Eq. 9).

\subsection{High-mass binaries}

In contrast with the original Kroupa IBP, for high-mass binaries, we adopt observational distributions derived for $\mathrm{O}$ and $\mathrm{B}-$ dwarfs to generate the birth population. Similarly to the original Kroupa IBP, no pre-main-sequence eigenevolution is applied in our modified version.

We adopt the distributions derived by Sana et al. (2012) who analysed the $\mathrm{O}$ star population of six nearby Galactic open stellar clusters. Note that the binarity found by Sana et al. (2012) is not 100 per cent and, since we assume, as usual, that star clusters are formed with a dominant population of binaries, i.e. with $\approx 100$ per cent of binaries, we should extend those distributions and normalise them in order to take this into account.

Following Oh et al. (2015), the period distribution is given by

$$
f_{P}=0.23 \times \log _{10}(P / \text { days })^{-0.55},
$$

where $\log _{10}(P /$ days $) \in[0.15,6.7]$. This distribution and range ensure that the cumulative binary fraction becomes unity.

In an analogous way, we extend the eccentricity distribution up to unity which results in the following distribution

$$
f_{e}=0.55 \times e^{-0.45}
$$

where $e \in[0.0,1.0]$.

Regarding the mass ratio, Sana et al. (2012) found a uniform distribution, i.e.

$$
f_{q}=1.0
$$


where $q \in[0.1,1.0]$.

We emphasize that the correct way of pairing birth low-mass and high-mass binaries and preserving the IMF is as follows. First, we generate an array of all stars (twice the number of binaries) from the IMF. Second, we select the more massive star (primary) from the array of stars, a mass ratio from the uniform distribution, and then compute the 'ideal' secondary mass. After that, the star that has the closest mass to the 'ideal' secondary mass is chosen from the array to be paired with the primary. Finally, both stars are removed from the array. Then, we proceed with the binary generation until the primary mass is smaller than $5 \mathrm{M}_{\odot}$. After that point, the above procedure remains the same with the exception that the secondary is chosen randomly in the array.

Finally, as done by Kroupa (1995b) for low-mass binaries, the upper envelope and circularization period in the plane $e$ vs. $\log _{10}(P)$ for high-mass binaries have to be consistent with to observational data. Here, we applied the criterion derived in Moe \& Di Stefano (2016) who considered observational data of O and B-dwarfs. This guarantees that the binary components do not fill their Roche-lobe by a factor greater than 70 per cent at the pericentre and it is achieved by the following restriction

$$
e_{\max }=1-\left(\frac{P}{P_{\text {circ }}}\right)^{-2 / 3},
$$

where $P>P_{\text {circ }}$, and the circularization period is given by $P_{\text {circ }}=$ 2 days.

In Fig. 2, we show the main distributions associated with the modified Kroupa IBP with respect to high-mass binaries, i.e. primary mass (top left-hand panel), mass ratio (top right-hand panel), period (bottom left-hand panel), and eccentricity (bottom right-hand panel). We also include the distributions of the original Kroupa IBP (Section 3.2) for comparison, as in Fig. 1.

We emphasize here that a way to determine the birth highmass binary distributions would be to investigate if the assumed hypothesis leads to the observed number of runaway massive stars through dynamical mass segregation to the cluster core and partner exchanges through dynamical encounters there between the massive stars (Kroupa 2008). This was partially done by Oh \& Kroupa (2016) who showed that the birth mass ratio distribution for O-star primaries must be near uniform for mass ratios greater than 0.1 , consistent with observational results of Sana et al. (2012). Finally, as massive stars require their own process of converting the birth population into the initial population, we assume here that such a process has already taken place and we adopt directly observational distributions, which is a reasonable approach considering the lack of understanding in this process. This is also consistent with the fact that this process should be very fast for massive stars (Railton et al. 2014).

\section{RESULTS}

In this section, we present the main results associated with the modified Kroupa IBP in a comparative way with the original Kroupa IBP. We show results for Galactic field late-type binaries and GCs. We end this section by showing that our modified Kroupa IBP solves the problem associated with present-day GC CMDs.

\subsection{Stimulated evolution}

In order to compare our results with Galactic field binaries, we assume as usual that Galactic field binaries come from the dissolution of dense star clusters, after stimulated evolution (Kroupa 1995b). Such a dissolution is assumed to be caused mainly by the residual gas removal due the evolution of massive stars (winds and, eventually, supernovas).

For the embedded clusters, we assume that initial clusters follow the Marks-Kroupa relation between half-mass radius and embedded mass of stars in the embedded cluster (Marks \& Kroupa 2012), i.e.

$$
\left(\frac{R_{h}}{\mathrm{pc}}\right)=0.1\left(\frac{M_{\mathrm{ecl}}}{\mathrm{M}_{\odot}}\right)^{0.13},
$$

where $R_{h}$ is the cluster initial half-mass radius and $M_{\text {ecl }}$ is the stellar mass in the embedded cluster.

Even though this is the most accurate approach, this is not doable yet with the current version of the MOCCA code, because the expansion of the embedded cluster due to residual gas removal, which takes place after few $10^{5}$ yr of cluster evolution (e.g. Banerjee \& Kroupa 2017), is not yet implemented in MOCCA.

In order to mimic this expansion and residual gas removal, we performed many numerical experiments to look for an initial expansion factor in the Marks-Kroupa relation to provide good agreement with observations. In order to find the best expansion factor, we assumed five different values, namely $1,2,5,7$, and 10, that should be multiplied by the half-mass radius in Eq. 16 to provide appropriate input for MOCCA models. In addition, since the stimulated evolution varies with the cluster mass, we also verify which expansion factor gives better results for combinations of stimulated evolution timescales and cluster mass. We assumed three different timescales, namely 1,5 and $10 \mathrm{Myr}$, and four different cluster masses, which is given in MOCCA by the number of objects (single stars + binaries), namely 10k, 20k, 50k and 100k. Note that these different timescales and cluster masses allow us to infer the strength of the dynamical processing in shaping the distributions through stimulated evolution.

All these models were evolved and compared with the observational mass-dependent binary fraction (e.g. Dorval et al. 2017, their Fig. 2) and properties of Galactic field late-type binaries (Section 7.2).

The best agreement with observations was achieved for an expansion factor of $\approx 2-4$, for a stimulated evolution timescale of $\approx 5$ Myr. This is also consistent with the results found by Pelupessy \& Portegies Zwart (2012) and Brinkmann et al. (2017) who showed, using direct $N$-body calculations, that embedded clusters expand by approximately this factor, if the star-formation efficiency is $\approx 1 / 3$, i.e. if $\approx 2 / 3$ of the initial gas is lost in the star cluster formation process. The properties of this MOCCA model are given in Table 3.

Assuming an expansion factor of $\approx 3$, the initial half-mass radius of MOCCA models should be

$$
\left(\frac{R_{h, \mathrm{MOCCA}}}{\mathrm{pc}}\right) \approx 0.3\left(\frac{M_{\mathrm{ecl}}}{\mathrm{M}_{\odot}}\right)^{0.13},
$$

Note that Marks et al. (2011) found that 50 per cent of all Galactic field binaries originate from clusters with $M_{\text {ecl }} \lesssim 300$ $\mathrm{M}_{\odot}$. This in principle might be a problem due to the fact that MOCCA models should contain at least 10k objects (single stars 
Table 3. Initial conditions of the best-fit model, which was used in the stimulated evolution, obtained by averaging over all 10 models. See Section 7.1, for more details.

\begin{tabular}{|c|c|}
\hline Mass $\left[\mathrm{M}_{\odot}\right]$ & $(9.23 \pm 0.16) \times 10^{3}$ \\
\hline Number of objects & $10^{4}$ \\
\hline Binary fraction $^{\mathrm{a}}[\%]$ & 95 \\
\hline Metallicity $\left[\mathrm{Z}_{\odot}\right]$ & 0.02 \\
\hline Central density $\left[\mathrm{M}_{\odot} \mathrm{pc}^{-3}\right]$ & $(1.5 \pm 0.5) \times 10^{4}$ \\
\hline Tidal radius $[\mathrm{pc}]$ & 26.0 \\
\hline Half-mass radius [pc] & $0.86 \pm 0.01$ \\
\hline Core radius $[\mathrm{pc}]$ & $0.28 \pm 0.01$ \\
\hline Galactocentric distance $[\mathrm{kpc}]$ & $8.01 \pm 0.07$ \\
\hline Half-mass radius relaxation time [Myr] & $32.5 \pm 0.7$ \\
\hline Central velocity dispersion $\left[\mathrm{km} \mathrm{s}^{-1}\right]$ & $5.1 \pm 0.7$ \\
\hline Duration of stimulated evolution [Myr] & $4.9 \pm 0.5$ \\
\hline
\end{tabular}

a We set the initial binary fraction different from 100 per cent in order to avoid computational problems that arise in MOCCA if there is no single star in the initial model.

+ binaries) which gives, in general, masses of one order of magnitude higher than that. However, in our experiments, models with $10 \mathrm{k}$ and $20 \mathrm{k}$ give similar results and the $10 \mathrm{k}$ model might be used as an upper limit associated with the influence of dynamics during stimulated evolution. Said that, we decided to show the results for a cluster of 10k which gives not only such an upper limit, but also is the closest possible MOCCA model to a low-mass embedded cluster from which the majority of Galactic field binaries originate. The validity of this assertion is demonstrated in the Appendix, in which we show that during the phase of the cluster expansion connected with residual gas removal, binaries with periods longer than $\approx 10^{7}$ days are modified by dynamical interactions. Therefore, only the tail of the observed period distribution can be modified by dynamics before the binaries become a part of the field population.

This MOCCA model is then dynamically equivalent for the binary population to the embedded clusters which have an expansion phase. We define 'dynamical equivalence' as follows: if two clusters with different masses and different initial radii dynamically evolve an identical initial binary population to similar distribution functions of binaries, then these two clusters are 'dynamically equivalent'. Cluster evolution need not be over the same time scales for dynamical equivalence to be reached.

For instance, when comparing the results for the models in Kroupa (1995b), composed initially of 200 binaries and computed for a few hundred Myr, with the models in Kroupa et al. (2001) composed of 5,000 binaries and integrated for a Myr, we notice that both clusters are dynamically equivalent. The corresponding binary distribution functions for these two clusters look very similar. They started with the same binary-star distributions and were evolved in very different cluster environments with and without stellar evolution (compare, for instance, fig. 7 in Kroupa (1995b) with figs. 10 and 11 in Kroupa et al. (2001)). In addition, Giersz et al. (2016) showed that MOCCA and N-body model binary distributions are evolved in a remarkably similar way, not only their period distributions (see their Fig. 2 which shows a comparison between MOCCA model and BIPoS code (Marks et al. 2011)), but also for binding energy, mass ratio and eccentricity distributions.

We emphasize here that dynamically equivalent models do not have exactly the same binary populations (i.e. same binary distributions) after stimulated evolution. However, they are very similar and with this concept it is possible to find general solutions to the class of dynamically equivalent clusters which lead to a very sim- ilar final binary population after finding one N-body solution (sec. 4.3 and sec. 6.4 in Kroupa (1995a) and sec. 4 in Kroupa (1995c)) ${ }^{4}$.

In order to further illustrate this important concept, we show in the Appendix B, as an example, that three different initial MOCCA models are dynamically equivalent over different stimulated evolution timescales by showing their resulting binary distributions (Fig. $\mathrm{B} 1)^{5}$.

All the above implies that our approach is reasonable and that MOCCA models represent clusters which have a dynamically equivalent history to the real embedded clusters which have undergone an expansion phase.

The stimulated evolution was performed as follows. We evolved for $\approx 5$ Myr two star cluster models with $M_{\mathrm{ecl}} \approx 10^{4} \mathrm{M}_{\odot}$ and 95 per cent of binaries that follow the original Kroupa IBP and the modified Kroupa IBP. For each cluster model, we performed 10 realizations in order to reduce fluctuation that arise from the small number of binaries in the clusters $(\approx 10 \mathrm{k})$.

In what follows, we show the results for our averaged (over 10 realizations) above-described models following Eq. 17.

\subsection{Galactic field late-type star distributions}

One of our goals, apart from the solution of the problems presented in Section 5 related to GC CMDs, is to not worsen the premain-sequence eigenevolution process with respect to late-type binaries in the Solar neighbourhood, i.e. eccentricity, period and mass ratio correlations, and for short-period binaries, a bell-shaped eccentricity distribution and a mass ratio distribution which rises towards unity.

These observational features were derived from long-term observations and here we compare both original and modified Kroupa IBPs with distributions extracted from the following studies ${ }^{6}$ :

- all G-dwarf period: DM91 (Duquennoy \& Mayor 1991),

- all M-dwarf period: FM92 (Fischer \& Marcy 1992),

- all G-dwarf eccentricity: DM91,

- short-period G-dwarf mass ratio: M1992 (Mazeh et al. 1992),

- long-period G-dwarf mass ratio: DM91,

- all M-dwarf mass ratio: B2010 (Bergfors et al. 2010),

- all late-type-dwarf mass ratio: RG97 (Reid \& Gizis 1997).

Note that we compare the mass ratio distribution of M-dwarf binaries with the data from Bergfors et al. (2010), that provide a similar distribution to that from Fischer \& Marcy (1992).

For G-dwarfs, we opted for not including the data from Raghavan et al. (2010) for the following reason. As already pointed out by Marks \& Kroupa (2011), Raghavan et al. (2010) found a mass-ratio distribution for binaries with a solar-type primary that is different from Duquennoy \& Mayor (1991). Since the range of

4 We would like to emphasize that using dynamical equivalence does not imply that a cluster evolves dynamically in the same way as another cluster. It merely means that, over a certain time scale, the dynamical evolution processes the binary population similarly.

5 We note that a demonstration of dynamical equivalence as defined here in the form of low-N dynamical simulations for the specific case studied in this work will be useful to further test this conjecture, whereby the small-N computations presented in Kroupa (1995a,b,c) are the basis of formulating it. There, the scaling from one dynamically-equivalent solution to another is also discussed.

6 Our definitions for the late-type binaries are as follows: all late-type, $\mathrm{G}$ and M-dwarfs have primary masses in the ranges $[0.08,2.0],[0.8,1.2]$, and $[0.08,0.6]$, respectively, all in units of $\mathrm{M}_{\odot}$. 
(a)
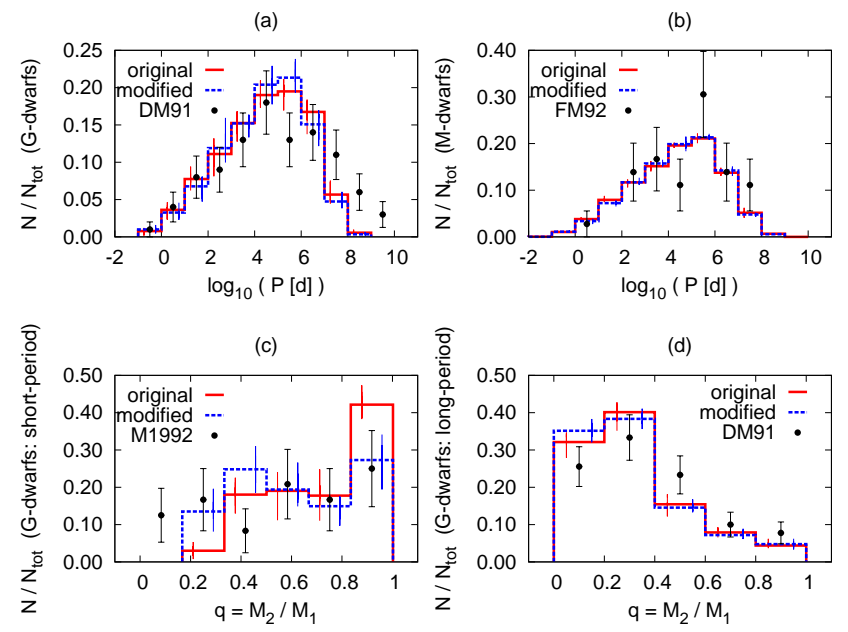

(e)
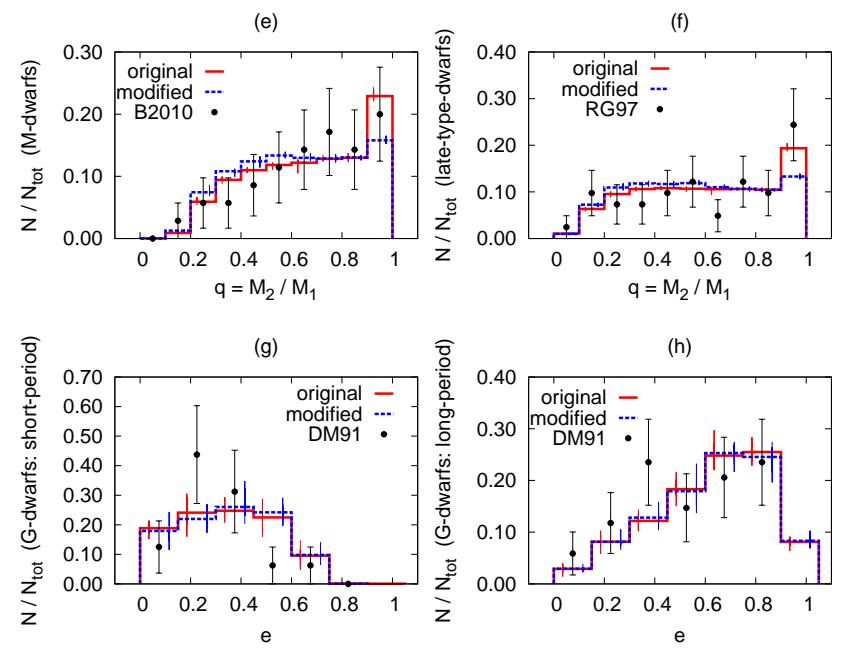

Figure 7. Comparison with Galactic field late-type binaries, after stimulated evolution, for an averaged model evolved with the original Kroupa IBP (Section 3, solid line) and an averaged model evolved with the modified Kroupa IBP (Section 6, dashed line). In panels (a) and (b), we plot the period distributions of $\mathrm{G}$ and $\mathrm{M}$-dwarfs respectively. In panels (c) and (d) we show the mass ratio distributions of short-period and long-period Gdwarfs, respectively. Mass ratio distributions for M-dwarfs and all dwarfs such that $M_{1}$ is smaller than $2 \mathrm{M}_{\odot}$ are shown in panels (e) and (f), respectively. Finally, in panels $(\mathrm{g})$ and $(\mathrm{h})$, we exhibit the eccentricity distributions for short-period and long-period G-dwarfs, respectively. Observational data is plotted with filled circles and were extracted from Duquennoy \& Mayor (1991) (DM91), Mazeh et al. (1992) (M1992), Fischer \& Marcy (1992) (FM92), Reid \& Gizis (1997) (RG97), and Bergfors et al. (2010) (B2010). Vertical lines for the IBPs correspond to the entire range of all 10 realizations outcomes, and for the data to Poisson errors. For more details, see Section 7.2.

primary masses in both works are similar, their mass-ratio distributions should be also similar if the periods are comparable. The reason for that is likely due to different approaches in both works. The survey performed by Duquennoy \& Mayor (1991) lasted 13 years and they found accurate orbital solutions for their binaries. On the other hand, Raghavan et al. (2010) compiled data from different sources and techniques. Given that, we preferred to compare our models with data from Duquennoy \& Mayor (1991) ${ }^{7}$.

7 See also the comments in Kroupa (2009).
Additional observational works could be also included in comparison. For instance, the results by Halbwachs et al. (2003) who investigated early-type F- and K-dwarf binaries and those by Fisher et al. (2005) who presented an incomplete survey of spectroscopic binaries. In order to use those results, one could apply the Kroupa IBP to this data set using stimulated evolution, but only by also modelling the complicated biases and selection effects, which is, however, out of scope of the present investigation. Finally, Rastegaev (2010) presented a survey of population II (sub-dwarf) stars. The authors note that the population II period distribution is narrower and biased towards shorter periods than the population I distribution (their Fig. 10). Even though analysing population II stars is not an objective here, it will be interesting to assume the universality hypothesis and then to investigate which population of star clusters may, if at all, account for the observed population II binary distributions. The mass function of metal-poor (population II) star clusters may have been different to that observed today for population I. In such an investigation, it will be necessary to model all selection effects and biases inherent to the analysis by Rastegaev (2010). A particular aspect of such a modelling will be the inclusion of population I stars and binaries which have acquired large proper motions, e.g. by being ejected out of their population I birth clusters. Such a contribution is likely to be found in a proper-motion selected sample in addition to population II, and may skew or alter the deduced binary star distribution functions. Again, modeling population II via the Kroupa IBP and stimulated evolution is not in the scope of this investigation, but is doable in future works.

In Fig. 7, we compare the observational distributions used in this work with our averaged models, after stimulated evolution, concerning the original Kroupa IBP and the modified Kroupa IBP. Vertical lines in the figure correspond to the entire range of all 10 realizations outcomes (for the IBPs), and to Poisson errors (for observational data).

Note that both IBPs provide good agreement with observations. In addition, both IBPs give similar distributions with the exception of the mass ratio distributions.

In panel (c), we show the mass ratio distribution of shortperiod binaries whose primaries are G-dwarfs. Note that the fraction of binaries with high mass ratio $q \gtrsim 0.9$ is reduced, this is expected given our initial motivation for changing the pre-mainsequence eigenevolution prescription.

In panel (e) and (f) we show mass ratio distributions of all binaries whose primaries are M-dwarfs and late-type-dwarfs, respectively. As for G-dwarfs, we also see a reduction in the fraction of high mass ratio binaries. This is again expected due to the revised pre-main-sequence eigenevolution derived here.

Finally, Fig. 7 clearly shows that none of the previous results achieved with the original Kroupa IBP are damaged when our modified Kroupa IBP is adopted. In other words, our modified Kroupa IBP is not only consistent with observational data of late-type binaries in the Galactic field, but also provides qualitatively similarly good description when compared with the original Kroupa IBP.

\subsection{Globular cluster colour-magnitude diagrams}

After showing that the modified Kroupa IBP provides good agreement with observational data of Galactic field binaries (i.e. our modifications do not worsen previously established constraints), we can turn to the solution of our initial problem.

In Section 5, we presented the motivation for our investigation showing the present-day CMDs and colour distributions derived from them of a set of six GC models with different initial and 
(1)

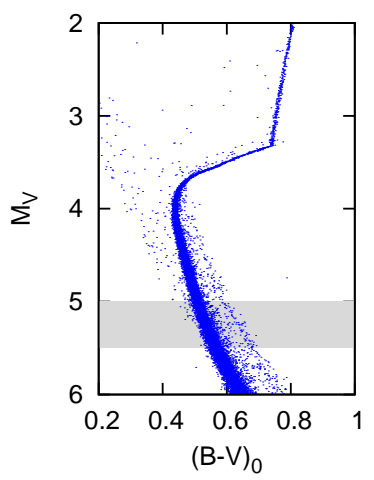

(3)

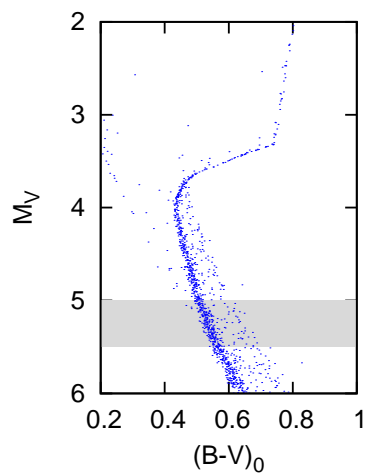

(5)

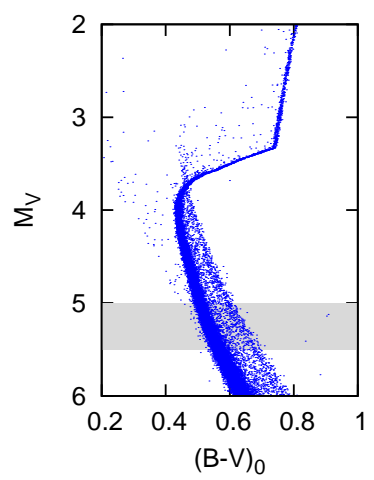

(2)

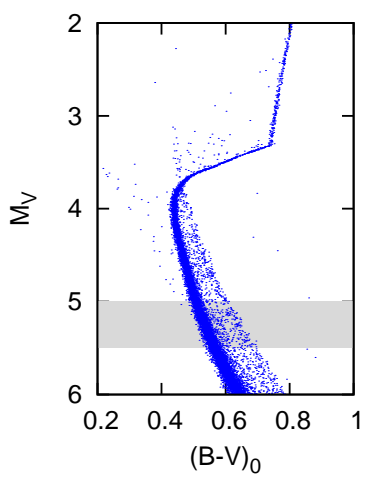

(4)

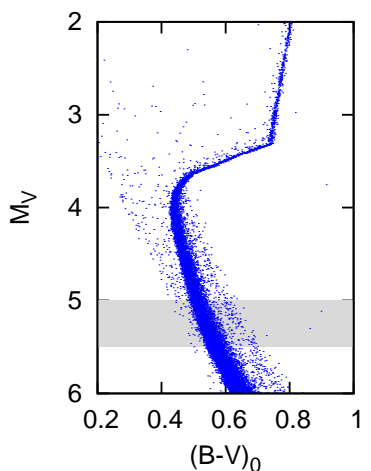

(6)

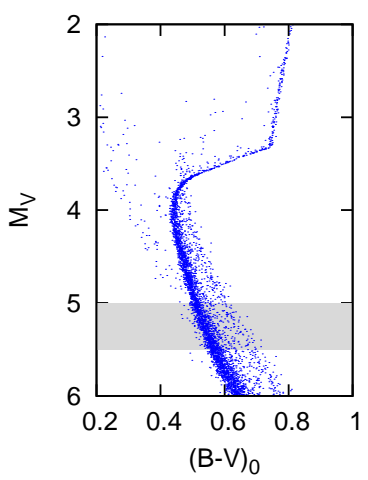

Figure 8. Present-day CMDs from simulated observations of the six GC models (Table 2) evolved with the modified Kroupa IBP (Section 6). The gray areas indicate the regions used to generate the colour distributions in Fig. 9. Note that there is no clear and pronounced binary sequence due to short-period low-mass binaries with mass ratios of unity in such CMDs, specially close to the turn-off. For more detail see Section 7.3.

present-day properties. All six models there were set with the original Kroupa IBP (Section 3). While comparing with observation of the nearest GCs (M4 and NGC6397), we showed that models set with the original Kroupa IBP are not able to predict realistic binary distributions in GCs. This is because it provides not only a clearly visible binary main-sequence (due to short-period low-mass binaries with mass ratios equal to unity) which is not seen in the CMDs of real GCs, but it also predicts CMD colour distributions below
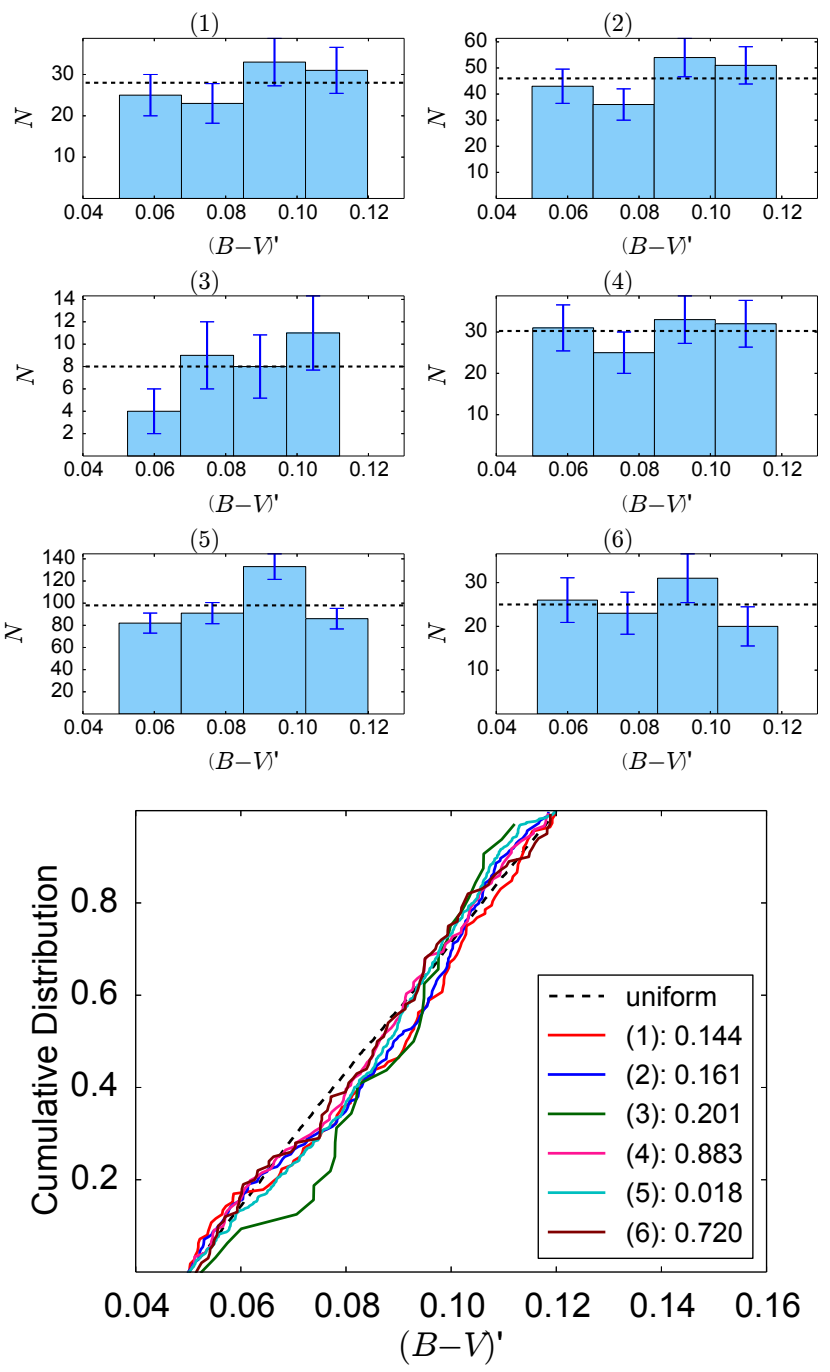

Figure 9. Colour distributions derived from the CMDs shown in Fig. 8, following the procedure described in Section 5.1. Notations as in 4. Only stars with $(B-V)^{\prime}>0.05$ are used to compute the above distributions. Note that in all colour distributions in the six models following the modified Kroupa IBP are are consistent uniform distributions. This is supported by the statistical test, which does not allows us to reject the hypothesis that they are uniform with more than $99 \%$ of confidence (see $p$-values). For more detail see Sections 7.3.

the turn-off peaked at the right edge of the distributions (associated again with high-q binaries).

We can at this point perform a similar analysis as done in Section 5, but taking into account our modified Kroupa IBP (Section 6). We thus simulate models with the same six initial cluster conditions and perform photometry of present-day models. The six model, now set with the modified Kroupa IBP, properties are shown in Table 2 and Fig. 8 exhibits their present-day CMDs.

Note that present-day GC model properties are similar for both set of models (compare with Table 1), which indicates that our modifications do not play a key role in the cluster dynamical evolution. However, while comparing Figs. 8 and 3 we do notice huge differences.

First, that pronounced and clearly visible binary sequence close and above the turn-off is not present in models set with the modified Kroupa IBP. Second, we do not clearly see binary se- 
quences below the turn-off associated with high-q binaries in all models. This is a result of our revised pre-main-sequence eigenevolution prescription which gives a mass-dependent strength of the changes to binary properties (Section 6.1). This leads to a reduced fraction of high mass ratio binaries as well as a uniform spread in the mass ratio in the range between 0.9 and 1.0, for those binaries that would have equal mass components.

Proceeding further, we generated colour distribution is the same way described in Section 5.1, which are shown in Fig. 9. Notice that all histograms seem to be consistent with uniform distributions, as in observations. Again, we applied one-sample Kolmogorov-Smirnov tests for uniformity. The $p$-values of these tests are displayed in the bottom panel of Fig. 9. The tests do not permit us to rule out the null hypothesis that they are uniform with more than 99 per cent of confidence, even though we can reject the null hypothesis for model 5 with more than 98 per cent of confidence.

We conclude then that our modifications to the Kroupa IBP bring present-day GC models closer to real GCs, which corresponds to a step forward towards a better prescription for initial cluster conditions.

We emphasize that the solution to the problem with respect to present-day GC CMDs (without worsening previous results) presented here open the possibility that a universal IBP might be at the origin of the binary population in both the Galactic field and in GCs. This is consistent with the conclusions reached by Leigh et al. (2015) and is important for a better understanding of the star formation process in proto-clusters.

\section{CONCLUSIONS AND PERSPECTIVE}

The original description of pre-main-sequence eigenevolution of a birth binary population by Kroupa (1995b), while being consistent with Galactic-field and open cluster binary-star data, is found here to lead to too many $q=1$ systems which implies that the CMDs of GCs ought to have pronounced binary sequences above the turn-off and to the right of the MS. This is not observed to be the case. In this contribution we revise th Kroupa pre-main-sequence eigenevolution model such that it is consistent with the Galacticfield, open cluster and GC data.

To achieve this, we assumed a mass-dependent strength for pre-main-sequence eigenevolution such that the lower the primary mass, the weaker the changes in the birth population. This procedure results in a smaller fraction of short-period binaries with equal mass components which provides qualitatively similarly good description of observations of Galactic field late-type binaries and GCs.

We also assumed distributions for massive binaries such that they follow directly distributions derived from observations of $\mathrm{O}$ dwarfs in open stellar clusters. This is consistent with the fact that pre-main-sequence timescales are extremely short for massive stars.

Finally, we emphasize that our modified Kroupa IBP should not change results achieved with the original Kroupa IBP with respect to young star clusters and GCs. In addition, this paper corresponds to the first step towards a better description of the IBP that should seed population synthesis codes (after dynamical population synthesis) and star cluster evolution codes, as well as a better understanding of clustered star formation processes such as energy and angular momentum redistribution within very young binary systems.
In following investigations of the modified Kroupa IBP, we will verify the influence of parameters that control binary stellar evolution such as the energy budget during the common-envelope phase and the angular momentum loss formalisms for interacting binaries, on populations of white dwarf-main sequence postcommon-envelope phase binaries and cataclysmic variables.

\section{ACKNOWLEDGEMENTS}

We would like to kindly thank Michael Marks for useful discussions and suggestions. We would also like to thank an anonymous referee for numerous comments and suggestions. DB was supported by the CAPES foundation, Brazilian Ministry of Education through the grant BEX 13514/13-0 and by the National Science Centre, Poland, through the grant UMO2016/21/N/ST9/02938. AA would like to acknowledge support from the National Science Centre, Poland, through the grant UMO2015/17/N/ST9/02573 and partial support from Nicolaus Copernicus Astronomical Centre's grant for young researchers.

\section{REFERENCES}

Askar A., Giersz M., Pych W., Dalessandro E., 2017a, preprint, (arXiv:1703.09160)

Askar A., Bianchini P., de Vita R., Giersz M., Hypki A., Kamann S., 2017b, MNRAS, 464, 3090

Banerjee S., Kroupa P., 2017, A\&A, 597, A28

Belloni D., Zorotovic M., Schreiber M. R., Leigh N. W. C., Giersz M., Askar A., 2017, MNRAS, 468, 2429

Bergfors C., et al., 2010, A\&A, 520, A54

Bonnell I., Bastien P., 1992, in McAlister H. A., Hartkopf W. I., eds, Astronomical Society of the Pacific Conference Series Vol. 32, IAU Colloq. 135: Complementary Approaches to Double and Multiple Star Research. p. 206

Bressert E., et al., 2010, MNRAS, 409, L54

Brinkmann N., Banerjee S., Motwani B., Kroupa P., 2017, A\&A, 600, A49

Dorval J., Boily C. M., Moraux E., Roos O., 2017, MNRAS, 465, 2198

Duchêne G., 1999, A\&A, 341, 547

Duquennoy A., Mayor M., 1991, A\&A, 248, 485

Fischer D. A., Marcy G. W., 1992, ApJ, 396, 178

Fisher J., Schröder K.-P., Smith R. C., 2005, MNRAS, 361, 495

Fregeau J. M., Cheung P., Portegies Zwart S. F., Rasio F. A., 2004, MNRAS, 352,1

Fukushige T., Heggie D. C., 2000, MNRAS, 318, 753

Giersz M., Heggie D. C., Hurley J. R., Hypki A., 2013, MNRAS, 431, 2184

Giersz M., Leigh N., Hypki A., Lützgendorf N., Askar A., 2015, MNRAS, 454,3150

Giersz M., Leigh N., Marks M., Hypki A., Askar A., 2016, in Meiron Y., Li S., Liu F.-K., Spurzem R., eds, IAU Symposium Vol. 312, Star Clusters and Black Holes in Galaxies across Cosmic Time. pp 213-222, doi:10.1017/S1743921315007838

Goodwin S. P., Kroupa P., 2005, A\&A, 439, 565

Halbwachs J. L., Mayor M., Udry S., Arenou F., 2003, A\&A, 397, 159

Harris W. E., 1996, AJ, 112, 1487

Hénon M. H., 1971, Astrophysics and Space Science, 14, 151

Hurley J. R., Pols O. R., Tout C. A., 2000, MNRAS, 315, 543

Hurley J. R., Tout C. A., Pols O. R., 2002, MNRAS, 329, 897

Hypki A., Giersz M., 2013, MNRAS, 429, 1221

King R. R., Goodwin S. P., Parker R. J., Patience J., 2012, MNRAS, 427, 2636

Kouwenhoven M. B. N., Brown A. G. A., Goodwin S. P., Portegies Zwart S. F., Kaper L., 2009, A\&A, 493, 979

Kroupa P., 1995a, MNRAS, 277, 1491

Kroupa P., 1995b, MNRAS, 277, 1507 
Kroupa P., 1995c, MNRAS, 277, 1522

Kroupa P., 2008, in Aarseth S. J., Tout C. A., Mardling R. A., eds, Lecture Notes in Physics, Berlin Springer Verlag Vol. 760, The Cambridge N-Body Lectures. p. 181 (arXiv:0803.1833), doi:10.1007/978-1-4020-8431-7_8

Kroupa P., 2009, A\&A, 500, 377

Kroupa P., 2011, in Alves J., Elmegreen B. G., Girart J. M., Trimble V., eds, IAU Symposium Vol. 270, Computational Star Formation. pp 141-149 (arXiv: 1012.1596), doi:10.1017/S1743921311000305

Kroupa P., Gilmore G., Tout C. A., 1991, MNRAS, 251, 293

Kroupa P., Aarseth S., Hurley J., 2001, MNRAS, 321, 699

Kroupa P., Weidner C., Pflamm-Altenburg J., Thies I., Dabringhausen J., Marks M., Maschberger T., 2013, The Stellar and Sub-Stellar Initial Mass Function of Simple and Composite Populations. p. 115, doi:10.1007/978-94-007-5612-0_4

Lada C. J., 2010, Phil. Trans. Roy. Soc. Lond. Series A, 368, 713

Lada C. J., Lada E. A., 2003, ARA\&A, 41, 57

Leigh N. W. C., Giersz M., Marks M., Webb J. J., Hypki A., Heinke C. O., Kroupa P., Sills A., 2015, MNRAS, 446, 226

Marks M., Kroupa P., 2011, MNRAS, 417, 1702

Marks M., Kroupa P., 2012, A\&A, 543, A8

Marks M., Kroupa P., Oh S., 2011, MNRAS, 417, 1684

Marks M., Janson M., Kroupa P., Leigh N., Thies I., 2015, MNRAS, 452, 1014

Mayer P., et al., 2017, A\&A, 600, A33

Mazeh T., Goldberg D., Duquennoy A., Mayor M., 1992, ApJ, 401, 265

Milone A. P., et al., 2012, A\&A, 540, A16

Moe M., Di Stefano R., 2016, preprint, (arXiv: 1606.05347 )

Oh S., Kroupa P., 2016, A\&A, 590, A107

Oh S., Kroupa P., Pflamm-Altenburg J., 2015, ApJ, 805, 92

Parker R. J., Meyer M. R., 2014, MNRAS, 442, 3722

Pelupessy F. I., Portegies Zwart S., 2012, MNRAS, 420, 1503

Pinsonneault M. H., Stanek K. Z., 2006, ApJ, 639, L67

Piotto G., et al., 2002, A\&A, 391, 945

Raghavan D., et al., 2010, ApJS, 190, 1

Railton A. D., Tout C. A., Aarseth S. J., 2014, Publ. Astron. Soc. Australia, 31, e 017

Rastegaev D. A., 2010, AJ, 140, 2013

Reid I. N., Gizis J. E., 1997, AJ, 113, 2246

Rubenstein E. P., Bailyn C. D., 1997, ApJ, 474, 701

Sadavoy S. I., Stahler S. W., 2017, MNRAS, 469, 3881

Sana H., et al., 2012, Science, 337, 444

Sarajedini A., et al., 2007, AJ, 133, 1658

Sollima A., Beccari G., Ferraro F. R., Fusi Pecci F., Sarajedini A., 2007, MNRAS, 380, 781

Spitzer L., 1987, Dynamical evolution of globular clusters

Stodółkiewicz J. S., 1986, Acta Astron., 36, 19

Wang L., et al., 2016, MNRAS, 458, 1450

\section{APPENDIX A: INFLUENCE OF DYNAMICAL ENCOUNTERS DURING STIMULATED EVOLUTION IN LOW- $N$ STAR CLUSTERS}

Here we show that from the point of view of IBP properties, star cluster models with $N=10,000$ objects are reasonable upper limits for low- $N$ star cluster models. For this purpose, we will make an order of magnitude estimate for the period of binaries which will certainly interact dynamically (probability of interaction equal to 1 ) with field stars during the time, $\Delta t$.

Let us consider a binary with semi major axis $a$, composed of stars with average stellar mass $\langle m\rangle$, which interacts with single stars with average mass, inside the half mass radius $R_{h}$. The probability of interaction is given by (e.g. Stodółkiewicz 1986, Eq. 30)

$$
\Pi=\pi p_{\mathrm{imp}}^{2} \eta v \Delta t
$$

where $\eta$ is the number density inside the half-mass radius, given by

$$
\eta=\frac{(1 / 2)(M /\langle m\rangle)}{(4 / 3) \pi R_{h}^{3}},
$$

where $\langle m\rangle=M / N$ (which is constant for a given IMF), being $N$ the number of objects (binaries + single stars) and $M$ the star cluster total mass, $v$ is the typical relative velocity, which is, assuming an isotropic velocity distribution, given by

$$
v^{2}=2 \sigma^{2}
$$

where $\sigma$ is the 3D velocity disperstion, given by (Spitzer 1987, Eq. 1-10)

$$
\sigma^{2} \approx 0.4 \frac{G M}{R_{h}}
$$

$p_{\text {imp }}$ is the impact parameter, given by (Spitzer 1987, Eq. 6-15)

$$
\begin{gathered}
p_{\text {imp }}^{2}=a^{2}\left(1+\frac{2 G M_{123}}{v^{2} a}\right), \\
\Leftrightarrow p_{\text {imp }}^{2} \approx 2 \frac{G M_{123} a}{v^{2}},
\end{gathered}
$$

because in embedded clusters, usually $\left(2 G M_{123}\right) /\left(v^{2} a\right) \gg 1$, where $M_{123}$ is the sum of the masses (binary mass plus single star mass).

Now, replacing the terms in Eq. A1 with Eqs. A2, A3, and A5, and assuming that only average stars take part in the interaction, i.e. $M_{123} \approx 3\langle m\rangle$, we have

$$
\Pi \approx 1.1 \frac{G^{1 / 2} M^{1 / 2}}{R_{h}^{5 / 2}} a \Delta t
$$

which gives, assuming that the star cluster follows the MarksKroupa relation (Eq. 17) between $R_{h}$ and $M$,

$$
\Pi \approx 1.17 \times 10^{-5}\left(\frac{M}{\mathrm{M}_{\odot}}\right)^{7 / 40}\left(\frac{a}{\mathrm{AU}}\right)\left(\frac{\Delta t}{\mathrm{Myr}}\right) .
$$

Equating the probability to unity we will get an estimation of the lower limit of the binary semimajor axis above which the binary will certainly interact with a field star during a time $\Delta t$, i.e.

$$
a \approx 8.55 \times 10^{4}\left(\frac{M}{\mathrm{M}_{\odot}}\right)^{-7 / 40}\left(\frac{\Delta t}{\mathrm{Myr}}\right)^{-1} .
$$

Now, from the Kepler's third law, we have

$$
\begin{gathered}
\left(\frac{P}{\mathrm{yr}}\right)^{2}=\left(\frac{a}{\mathrm{AU}}\right)^{3}\left(\frac{M_{12}}{\mathrm{M}_{\odot}}\right)^{-1} \\
\left(\frac{P}{\mathrm{~d}}\right) \approx 9.13 \times 10^{9}\left(\frac{M}{\mathrm{M}_{\odot}}\right)^{-21 / 80}\left(\frac{\Delta t}{\mathrm{Myr}}\right)^{-3 / 2},
\end{gathered}
$$


Table B1. Initial conditions of the three models used to show that the principle of dynamical equivalence is valid. As in previous simulations, for all three models, we assumed solar metallicity and set the initial binary fraction as 95 per cent. The IBP in all models is the modified Kroupa IBP. Notice that the three models have very distinct initial conditions, especially masses and half-mass radius relaxation times.

\begin{tabular}{lccc}
\hline \hline & \multicolumn{3}{c}{ Model } \\
\hline Property & $10 \mathrm{k}$ & $50 \mathrm{k}$ & $100 \mathrm{k}$ \\
\hline Mass $\left[\times 10^{4} \mathrm{M}_{\odot}\right]$ & 0.89 & 4.60 & 9.11 \\
Number of objects $\left[\times 10^{4}\right]$ & 1 & 5 & 10 \\
Central density [ $\times 10^{4} \mathrm{M}_{\odot} \mathrm{pc}^{-3}$ ] & 1.1 & 2.6 & 5.3 \\
Tidal radius [pc] & 25.8 & 44.1 & 55.6 \\
Half-mass radius [pc] & 0.83 & 1.05 & 1.14 \\
Core radius [pc] & 0.28 & 0.35 & 0.30 \\
Galactocentric distance $[\mathrm{kpc}]$ & 8.0 & 7.9 & 7.9 \\
Half-mass radius relaxation time $[\mathrm{Myr}]$ & 30.7 & 75.2 & 109.2 \\
Central velocity dispersion $\left[\mathrm{km} \mathrm{s}{ }^{-1}\right]$ & 4.3 & 11.1 & 16.1 \\
Duration of stimulated evolution [Myr] & 10.8 & 2.5 & 1.3 \\
\hline \hline
\end{tabular}

where $M_{12} \approx 2\langle m\rangle \approx 1 \mathrm{M}_{\odot}$ is the average binary mass, and $P$ is the period.

Finally, let us consider three cluster masses, namely $10^{2} \mathrm{M}_{\odot}$, $10^{3} \mathrm{M}_{\odot}$, and $10^{4} \mathrm{M}_{\odot}$, and estimate the minimum period for interactions in each of these clusters, during stimulated evolution $(\Delta t \approx 5 \mathrm{Myr})$ :

- $M \approx 10^{4} \mathrm{M}_{\odot} \Leftrightarrow N \approx 10^{4}: P \approx 7.3 \times 10^{7}$ days,

- $M \approx 10^{3} \mathrm{M}_{\odot} \Leftrightarrow N \approx 10^{3}: P \approx 1.3 \times 10^{8}$ days,

- $M \approx 10^{2} \mathrm{M}_{\odot} \Leftrightarrow N \approx 10^{2}: P \approx 2.4 \times 10^{8}$ days.

Notice that the smaller the number of objects in the cluster is (or the cluster mass), the longer is the minimum period for interactions. This implies that for low- $N$ clusters, mainly long-period binaries are affected by dynamical interactions. Note also that we assumed many simplifications in this calculation, which leads to an overestimation of such a minimum period. In reality, clusters are denser in the central parts and composed of objects with different masses, which leads to larger probabilities of binary interactions and in turn to smaller semimajor axis. Said that, the minimum period is likely to be shorter than that derived here. However, we do not expect substantial (many orders of magnitude) reduction of the estimated minimum period. This means that dynamical interactions between binaries and other objects during stimulated evolution will not have any influence on the bulk of binaries. Only the long-period tail of the distribution can be slightly changed. This leads us to the conclusion that very-low- $N$ clusters are dynamically equivalent (Kroupa 1995b) to our cluster model with $N=10^{4}$.

\section{APPENDIX B: DYNAMICAL EQUIVALENCE}

Here we show that the principle of dynamical equivalence associated with the stimulated evolution is valid. As stated in Section 7.1 , if two clusters with different masses and different initial characteristic radii dynamically evolve an identical initial binary population to similar distribution functions of binaries after stimulated evolution, then these two clusters are 'dynamically equivalent'. In order to show that we can find star cluster models that are dynamically equivalent, even for different initial conditions, we simulated evolution of three different cluster models with the MOCCA code. Properties of those models are summarized in Table B1.
We notice that, since two clusters with different initial conditions have different dynamical histories, in order to have an equivalent evolution, the timescale of the evolution has to be different. Indeed, this is shown in Fig. B1 where we depict the period, mass ratio, eccentricity and binding energy distributions associated with all binaries in the three clusters, initially and after stimulated evolution. As it is well known, when we evolve the three different initial models over the same physical time, their binary distributions will differ. However, if we control the stimulated evolution timescale, we can find a value such that their distributions will be similar after that time. In the particular case of the three models described in Table B1, if the timescales for $10 \mathrm{k}, 50 \mathrm{k}$ and $100 \mathrm{k}$ models are close to $10.8,2.5$ and $1.3 \mathrm{Myr}$, respectively, then all three clusters are dynamically equivalent. In general, there are pragmatically many combinations of stimulated evolution timescales such that these particular three clusters are dynamically equivalent and the combination above is just one example.

This simple exercise illustrates the principle of dynamical equivalence and its application, i.e. we can always find dynamical equivalent solutions to the problem of initial conditions, provided that each cluster evolves at its own particular timescale.

This paper has been typeset from a $\mathrm{T}_{\mathrm{E}} \mathrm{X} / \mathrm{LT} \mathrm{E} \mathrm{X}$ file prepared by the author. 

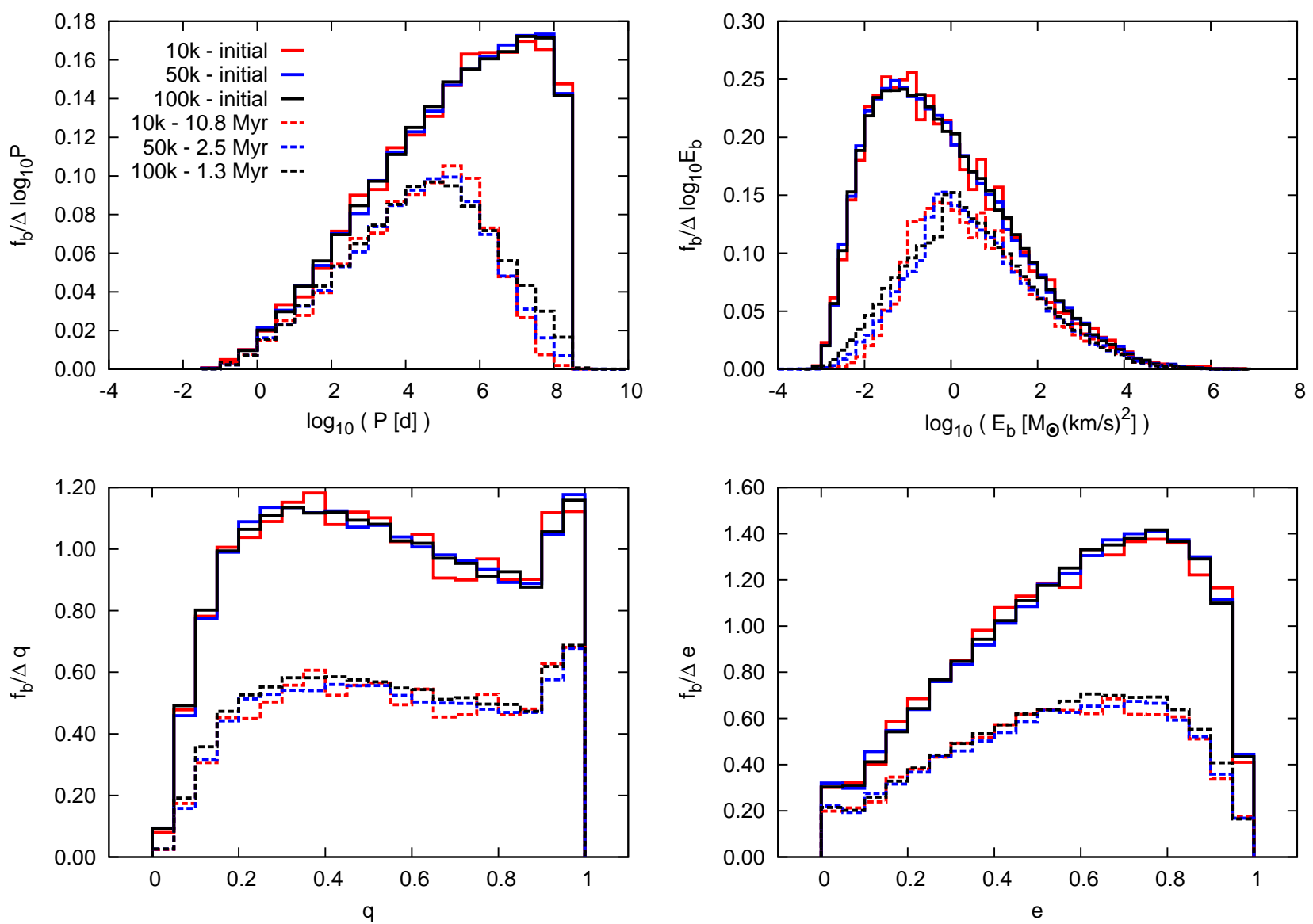

Figure B1. Distribution of periods (top left-hand panel), binding energies (top right-hand panel), mass ratios (bottom left-hand panel) and eccentricities (bottom right-hand panel) for all binaries. We depict initial properties as well as properties after stimulated evolution. The area under each distribution equals the total binary fraction. Notice that the distributions after stimulated evolution are remarkably consistent with each other. In addition, each cluster evolutionary timescale is different, which is needed for dynamical equivalence amongst them, since they have different initial properties. The prime lesson from this figure is that different initial cluster conditions can be evolved into similar binary distribution functions, provided that they evolve during different timescales. For more details see Appendix B. 